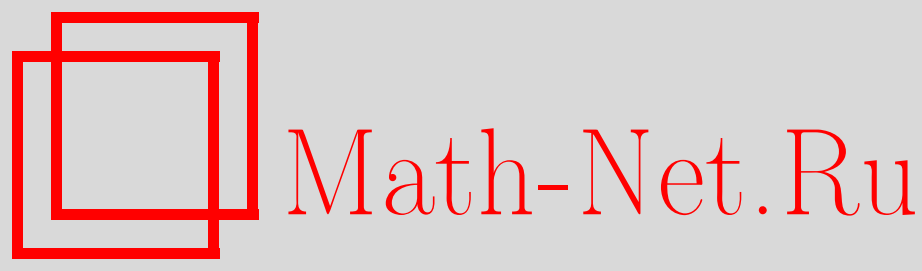

С. Э. Деркачёв, В. П. Спиридонов, Конечномерные представления эллиптического модулярного дубля, TMФ, 2015, том 183, номер 2, 177-201

DOI: https://doi.org/10.4213/tmf8817

Использование Общероссийского математического портала Math-Net.Ru подразумевает, что вы прочитали и согласны с пользовательским соглашением http://www.mathnet.ru/rus/agreement

Параметры загрузки:

IP : 34.227 .88 .159

26 апреля 2023 г., 13:54:19

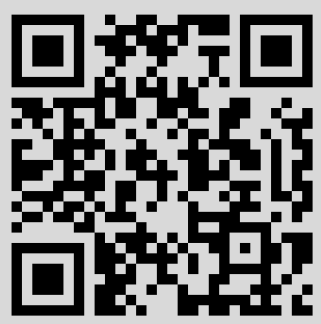




\title{
ФИЗИКА
}

Том 183, № 2

май, 2015

(C) 2015 г. $\quad$ С.Э. Деркачёв* ${ }^{*}$ В.П. Спиридонов ${ }^{\dagger}$

\section{КОНЕЧНОМЕРНЫЕ ПРЕДСТАВЛЕНИЯ ЭЛЛИПТИЧЕСКОГО МОДУЛЯРНОГО ДУБЛЯ}

\begin{abstract}
Изучается ядро интегрального оператора $\mathrm{M}(g)$, который зависит от "спина" $g$ и описывает некоторое эллиптическое преобразование Фурье. Оператор $\mathrm{M}(g)$ является сплетающим для эллиптического модулярного дубля, образованного из пары алгебр Склянина с параметрами $\eta$ и $\tau, \operatorname{Im} \tau>0, \operatorname{Im} \eta>0$. Для двумерных решеток $g=n \eta+m \tau / 2$ и $g=1 / 2+n \eta+m \tau / 2$ с несоизмеримыми величинами $1,2 \eta, \tau$ и с целыми числами $n, m>0$ оператор $\mathrm{M}(g)$ имеет конечномерное ядро, состоящее из произведений тета-функций с двумя различными модулярными параметрами, которое инвариантно относительно действия генераторов эллиптического модулярного дубля.
\end{abstract}

Ключевые слова: уравнение Янга-Бакстера, эллиптический модулярный дубль, эллиптические гипергеометрические функции.

DOI: $10.4213 / \operatorname{tmf} 8817$

Людвигу Фаддееву в честь восъмидесятилетия

\section{1. ЭЛЛИПТИЧЕСКИЙ МОДУЛЯРНЫЙ ДУБЛЬ}

Концепция модулярного дубля квантовой группы была предложена Фаддеевым в работе [1], в которой было показано, что квантовая алгебра $U_{q}\left(s l_{2}\right)$ с параметром деформации $q$ не определяет единственным образом пространство представлений и требует расширения. Такое расширение образуется двумя наборами генераторов $\mathbf{E}, \mathbf{F}, \mathbf{K}$ и $\widetilde{\mathbf{E}}, \widetilde{\mathbf{F}}, \widetilde{\mathbf{K}}$. Обычные алгебраические соотношения

$$
[\mathbf{E}, \mathbf{F}]=\frac{\mathbf{K}^{2}-\mathbf{K}^{-2}}{q-q^{-1}}, \quad \mathbf{K E}=q \mathbf{E K}, \quad \mathbf{K F}=q^{-1} \mathbf{F K},
$$

где $q=e^{\pi i \sigma}$, дополняются аналогичными соотношениями для операторов $\widetilde{\mathbf{E}}, \underset{\mathbf{F}}{\widetilde{\mathbf{K}}}$ с параметром $q$, замененным на $\tilde{q}=e^{\pi i / \sigma}$. Генераторы $\mathbf{E}, \mathbf{F}$ коммутируют с $\widetilde{\mathbf{E}}, \widetilde{\mathbf{F}}$.

${ }^{*}$ Санкт-Петербургское отделение Математического института им. В. А. Стеклова РАН, Санкт-Петербург, Россия. E-mail: derkach@pdmi.ras.ru

† Объединенный институт ядерных исследований, Лаборатория теоретической физики им. Н.Н. Боголюбова, Дубна, Московская обл., Россия., Дубна, Московская обл., Россия. E-mail: spiridon@theor.jinr.ru 
Генератор К антикоммутирует с $\widetilde{\mathbf{E}}, \widetilde{\mathbf{F}}$, а $\widetilde{\mathbf{K}}$ антикоммутирует с $\mathbf{E}, \mathbf{F}$. Эта алгебра обладает двумя центральными элементами - операторами Казимира. Первый из них имеет вид

$$
\mathbf{C}=\left(q-q^{-1}\right)^{2} \mathbf{F E}-q \mathbf{K}^{2}-q^{-1} \mathbf{K}^{-2}+2,
$$

а второй строится из операторов $\widetilde{\mathbf{E}}, \widetilde{\mathbf{F}}, \widetilde{\mathbf{K}}$ и $\tilde{q}$; его явный вид аналогичен (2). Специальные представления этого модулярного дубля были рассмотрены в работах [1]-[2].

Квантовые алгебры появились из теории уравнения Янга-Бакстера (ЯБ)

$$
\mathbb{R}_{12}(u-v) \mathbb{R}_{13}(u) \mathbb{R}_{23}(v)=\mathbb{R}_{23}(v) \mathbb{R}_{13}(u) \mathbb{R}_{12}(u-v),
$$

которое играет важную роль в математической физике [7]-[9]. Операторы $\mathbb{R}_{j k}$ действуют в подпространстве $\mathbb{V}_{j} \otimes \mathbb{V}_{k}$ тензорного произведения $\mathbb{V}_{1} \otimes \mathbb{V}_{2} \otimes \mathbb{V}_{3}$ трех (в общем случае различных) пространств $\mathbb{V}_{j}$. Переменные $u$ и $v$ называются спектральными параметрами. Алгебра Склянина [10], [11] является однопараметрической деформацией алгебры $U_{q}\left(s l_{2}\right)$ или эллиптической деформацией алгебры $s l_{2}$. Она возникает из уравнения $(3)$ в случае, когда $\mathbb{R}_{12}(u)$ задается $(4 \times 4)-R$-матрицей Бакстера [7],

$$
\mathbb{R}_{12}(u)=\sum_{a=0}^{3} w_{a}(u) \sigma_{a} \otimes \sigma_{a}, \quad w_{a}(u)=\frac{\theta_{a+1}(u+\eta \mid \tau)}{\theta_{a+1}(\eta \mid \tau)},
$$

и $\mathbb{R}_{13}(u), \mathbb{R}_{23}(v)$ являются $(2 \times 2)$-матрицами, заданными как копии следующего $L$-оператора:

$$
\mathrm{L}(u):=\sum_{a=0}^{3} w_{a}(u) \sigma_{a} \otimes \mathbf{S}^{a}=\left(\begin{array}{cc}
w_{0}(u) \mathbf{S}^{0}+w_{3}(u) \mathbf{S}^{3} & w_{1}(u) \mathbf{S}^{1}-i w_{2}(u) \mathbf{S}^{2} \\
w_{1}(u) \mathbf{S}^{1}+i w_{2}(u) \mathbf{S}^{2} & w_{0}(u) \mathbf{S}^{0}-w_{3}(u) \mathbf{S}^{3}
\end{array}\right),
$$

где $\sigma_{a}$ обозначает матрицы Паули и $\eta, \tau$ являются свободными параметрами, $\operatorname{Im} \tau>0$. Здесь $\theta_{a}(u \mid \tau)$ - тета-функции Якоби,

$$
\begin{array}{ll}
\theta_{1}(z \mid \tau)=-\sum_{n \in \mathbb{Z}} e^{\pi i(n+1 / 2)^{2} \tau} e^{2 \pi i(n+1 / 2)(z+1 / 2)}, & \theta_{2}(z \mid \tau)=\theta_{1}\left(z+\frac{1}{2} \mid \tau\right), \\
\theta_{3}(z \mid \tau)=e^{\pi i \tau / 4+\pi i z} \theta_{2}\left(z+\frac{\tau}{2} \mid \tau\right), & \theta_{4}(z \mid \tau)=\theta_{3}\left(z+\frac{1}{2} \mid \tau\right) .
\end{array}
$$

Удобно ввести базисные переменные $p=e^{2 \pi i \tau}$ и $q=e^{4 \pi i \eta}$. Тогда

$$
\theta_{1}(z \mid \tau)=\frac{e^{-\pi i z} \theta\left(e^{2 \pi i z} ; p\right)}{\mathrm{R}(\tau)}, \quad \mathrm{R}(\tau)=\frac{p^{-1 / 8}}{i(p ; p)_{\infty}},
$$

где

$$
\theta(t ; p)=(t ; p)_{\infty}\left(p t^{-1} ; p\right)_{\infty}, \quad(t ; p)_{\infty}=\prod_{k=0}^{\infty}\left(1-t p^{k}\right) .
$$

Тета-функции квазипериодичны, например

$$
\theta_{1}(z+1 \mid \tau)=-\theta_{1}(z \mid \tau), \quad \theta_{1}(z+\tau \mid \tau)=-e^{-2 \pi i z-\pi i \tau} \theta_{1}(z \mid \tau) .
$$

В дальнейшем нам потребуются общие соотношения

$$
\theta_{a}(z+m \tau \mid \tau)=\mu_{a} e^{-\pi i \tau m^{2}-2 \pi i m z} \theta_{a}(z \mid \tau), \quad m \in \mathbb{Z},
$$

где $\mu_{a}=(-1)^{m}$ для $a=1,4$ и $\mu_{a}=1$ для $a=2,3$. 
Уравнение ЯБ, соответствующее операторам (4) и (5), принимает вид $R L L$-соотношения

$$
\mathbb{R}_{12}(u-v) \mathrm{L}_{1}(u) \mathrm{L}_{2}(v)=\mathrm{L}_{2}(v) \mathrm{L}_{1}(u) \mathbb{R}_{12}(u-v),
$$

которое приводит к следующей алгебре Склянина [10]:

$$
\begin{aligned}
\mathbf{S}^{\alpha} \mathbf{S}^{\beta}-\mathbf{S}^{\beta} \mathbf{S}^{\alpha} & =i\left(\mathbf{S}^{0} \mathbf{S}^{\gamma}+\mathbf{S}^{\gamma} \mathbf{S}^{0}\right), \\
\mathbf{S}^{0} \mathbf{S}^{\alpha}-\mathbf{S}^{\alpha} \mathbf{S}^{0} & =i \mathbf{J}_{\beta \gamma}\left(\mathbf{S}^{\beta} \mathbf{S}^{\gamma}+\mathbf{S}^{\gamma} \mathbf{S}^{\beta}\right),
\end{aligned}
$$

где триплет $(\alpha, \beta, \gamma)$ является произвольной циклической перестановкой $(1,2,3)$. Структурные постоянные $\mathbf{J}_{\alpha \beta}$ не являются независимыми переменными и удовлетворяют ограничению

$$
\mathbf{J}_{12}+\mathbf{J}_{23}+\mathbf{J}_{31}+\mathbf{J}_{12} \mathbf{J}_{23} \mathbf{J}_{31}=0 \text {. }
$$

Параметризуя их как $\mathbf{J}_{\alpha \beta}=\left(\mathbf{J}_{\beta}-\mathbf{J}_{\alpha}\right) / \mathbf{J}_{\gamma}$ для $\gamma \neq \alpha, \beta$, что позволяет автоматически удовлетворить условие (9), можно написать

$$
\mathbf{J}_{1}=\frac{\theta_{2}(2 \eta \mid \tau) \theta_{2}(0 \mid \tau)}{\theta_{2}^{2}(\eta \mid \tau)}, \quad \mathbf{J}_{2}=\frac{\theta_{3}(2 \eta \mid \tau) \theta_{3}(0 \mid \tau)}{\theta_{3}^{2}(\eta \mid \tau)}, \quad \mathbf{J}_{3}=\frac{\theta_{4}(2 \eta \mid \tau) \theta_{4}(0 \mid \tau)}{\theta_{4}^{2}(\eta \mid \tau)}
$$

Постоянные $\mathbf{J}_{\alpha}$ также не являются независимыми, поскольку они параметризуются только двумя комплексными переменными $\eta$ и $\tau$.

Имеются два оператора Казимира, коммутирующие со всеми генераторами:

$$
\begin{gathered}
\mathbf{K}_{0}=\sum_{a=0}^{3} \mathbf{S}^{a} \mathbf{S}^{a}, \quad \mathbf{K}_{2}=\sum_{\alpha=1}^{3} \mathbf{J}_{\alpha} \mathbf{S}^{\alpha} \mathbf{S}^{\alpha}, \\
{\left[\mathbf{K}_{0}, \mathbf{S}^{a}\right]=\left[\mathbf{K}_{2}, \mathbf{S}^{a}\right]=0 .}
\end{gathered}
$$

Операторы $\mathbf{S}^{a}$ могут быть реализованы как конечноразностные операторы, действующие на функции комплексной переменной $z$ [11]:

$$
\begin{aligned}
\mathbf{S}^{a} & =e^{\pi i z^{2} / \eta} \frac{i^{\delta_{a, 2}} \theta_{a+1}(\eta \mid \tau)}{\theta_{1}(2 z \mid \tau)}\left[\theta_{a+1}(2 z-g+\eta \mid \tau) e^{\eta \partial_{z}}-\theta_{a+1}(-2 z-g+\eta \mid \tau) e^{-\eta \partial_{z}}\right] e^{-\pi i z^{2} / \eta}= \\
& =f_{a}(z) e^{\eta \partial_{z}}+f_{a}(-z) e^{-\eta \partial_{z}}
\end{aligned}
$$

где

$$
f_{a}(z)=e^{-\pi i \eta-2 \pi i z} i^{\delta_{a, 2}} \theta_{a+1}(\eta \mid \tau) \frac{\theta_{a+1}(2 z-g+\eta \mid \tau)}{\theta_{1}(2 z \mid \tau)}
$$

и $e^{ \pm \eta \partial_{z}}$ обозначают операторы сдвига, $e^{ \pm \eta \partial_{z}} \psi(z)=\psi(z \pm \eta)$. Переменная $g$ обычно задается как $g=\eta(2 \ell+1)$, и параметр $\ell \in \mathbb{C}$ называется спином. Мы также назовем $g$ спином. Параметры $\tau, \eta$ и $g$ характеризуют представления алгебры Склянина, поскольку они фиксируют значения операторов Казимира. Заметим, что наши операторы (11) отличаются от стандартных выражений умножением на экспоненты $e^{ \pm \pi i z^{2} / \eta}$ слева и справа (такой выбор приводит к аналитичности по переменной $e^{2 \pi i z}$ для сплетающего оператора, см. работу [12] и рассуждения ниже).

Эллиптический модулярный дубль был предложен в работе [13]. Определенная реализация этой алгебры вырождается в специальном пределе в модулярный дубль 
Фаддеева [1]. Интересующий нас дубль генерируется операторами $\mathbf{S}^{a}$ и новыми операторами $\widetilde{\mathbf{S}}^{a}$, которые удовлетворяют соотношениям

$$
\begin{aligned}
\widetilde{\mathbf{S}}^{\alpha} \widetilde{\mathbf{S}}^{\beta}-\widetilde{\mathbf{S}}^{\beta} \widetilde{\mathbf{S}}^{\alpha}=i\left(\widetilde{\mathbf{S}}^{0} \widetilde{\mathbf{S}}^{\gamma}+\widetilde{\mathbf{S}}^{\gamma} \widetilde{\mathbf{S}}^{0}\right), \\
\widetilde{\mathbf{S}}^{0} \widetilde{\mathbf{S}}^{\alpha}-\widetilde{\mathbf{S}}^{\alpha} \widetilde{\mathbf{S}}^{0}=i \widetilde{\mathbf{J}}_{\beta \gamma}\left(\mathbf{S}^{\beta} \mathbf{S}^{\gamma}+\mathbf{S}^{\gamma} \mathbf{S}^{\beta}\right),
\end{aligned}
$$

где триплет $(\alpha, \beta, \gamma)$ является произвольной циклической перестановкой $(1,2,3)$. Новые структурные константы с тильдой имеют аналогичную параметризацию $\tilde{\mathbf{J}}_{\alpha \beta}=\left(\tilde{\mathbf{J}}_{\beta}-\tilde{\mathbf{J}}_{\alpha}\right) / \tilde{\mathbf{J}}_{\gamma}$ для $\gamma \neq \alpha, \beta$, где

$$
\tilde{\mathbf{J}}_{1}=\frac{\theta_{2}(\tau \mid 2 \eta) \theta_{2}(0 \mid 2 \eta)}{\theta_{2}^{2}\left(\frac{\tau}{2} \mid 2 \eta\right)}, \quad \tilde{\mathbf{J}}_{2}=\frac{\theta_{3}(\tau \mid 2 \eta) \theta_{3}(0 \mid 2 \eta)}{\theta_{3}^{2}\left(\frac{\tau}{2} \mid 2 \eta\right)}, \quad \tilde{\mathbf{J}}_{3}=\frac{\theta_{4}(\tau \mid 2 \eta) \theta_{4}(0 \mid 2 \eta)}{\theta_{4}^{2}\left(\frac{\tau}{2} \mid 2 \eta\right)} .
$$

Отметим, что постоянные $\tilde{\mathbf{J}}_{\alpha}$ не являются независимыми от $\mathbf{J}_{\alpha}$. Униформизации (10) и (13) задают неявный вид этой зависимости, было бы интересно описать ее чисто алгебраическим или геометрическим способом.

Имеются два оператора Казимира, коммутирующие со всеми генераторами:

$$
\begin{gathered}
\widetilde{\mathbf{K}}_{0}=\sum_{a=0}^{3} \widetilde{\mathbf{S}}^{a} \widetilde{\mathbf{S}}^{a}, \quad \widetilde{\mathbf{K}}_{2}=\sum_{\alpha=1}^{3} \tilde{\mathbf{J}}_{\alpha} \widetilde{\mathbf{S}}^{\alpha} \widetilde{\mathbf{S}}^{\alpha}, \\
{\left[\widetilde{\mathbf{K}}_{0}, \widetilde{\mathbf{S}}^{a}\right]=\left[\widetilde{\mathbf{K}}_{2}, \widetilde{\mathbf{S}}^{a}\right]=0 .}
\end{gathered}
$$

Кросс-коммутационные соотношения между $\mathbf{S}^{a}$ и $\widetilde{\mathbf{S}}^{a}$ имеют вид

$$
\begin{array}{lll}
\mathbf{S}^{a} \widetilde{\mathbf{S}}^{b}=\widetilde{\mathbf{S}}^{b} \mathbf{S}^{a} & \text { при } & a, b \in\{0,3\} \quad \text { или } \quad a, b \in\{1,2\}, \\
\mathbf{S}^{a} \widetilde{\mathbf{S}}^{b}=-\widetilde{\mathbf{S}}^{b} \mathbf{S}^{a} & \text { при } & a \in\{0,3\}, b \in\{1,2\} \quad \text { или } \quad a \in\{1,2\}, b \in\{0,3\} .
\end{array}
$$

Следовательно, операторы $\mathbf{K}_{0}, \mathbf{K}_{2}$ коммутируют с $\widetilde{\mathbf{S}}^{b}$ и, наоборот, операторы $\widetilde{\mathbf{K}}_{0}$, $\widetilde{\mathbf{K}}_{2}$ коммутируют с $\mathbf{S}^{b}$, т. е. мы имеем четыре оператора Казимира:

$$
\left[\mathbf{K}_{0}, \widetilde{\mathbf{S}}^{a}\right]=\left[\mathbf{K}_{2}, \widetilde{\mathbf{S}}^{a}\right]=\left[\widetilde{\mathbf{K}}_{0}, \mathbf{S}^{a}\right]=\left[\widetilde{\mathbf{K}}_{2}, \mathbf{S}^{a}\right]=0 .
$$

Подобно алгебре Склянина эллиптический модулярный дубль является ассоциативной алгеброй. Благодаря некоммутативности генераторов $\mathbf{S}^{a}$ и $\widetilde{\mathbf{S}}^{a}$ этот дубль не является прямым произведением двух алгебр Склянина, хотя достаточно легко проследить разницу действий генераторов подалгебр на модули в различных порядках.

Имеется очевидная реализация генераторов второй алгебры Склянина конечноразностными операторами. А именно, операторы $\widetilde{\mathbf{S}}^{a}$ получаются из $\mathbf{S}^{a}$ простой перестановкой $2 \eta$ и $\tau$ (что требует $\operatorname{Im} \eta>0$ ):

$$
\begin{aligned}
\widetilde{\mathbf{S}}^{a}= & e^{2 \pi i z^{2} / \tau} \frac{i^{\delta_{a, 2}} \theta_{a+1}\left(\frac{\tau}{2} \mid 2 \eta\right)}{\theta_{1}(2 z \mid 2 \eta)} \times \\
& \times\left[\theta_{a+1}\left(2 z-g+\frac{\tau}{2} \mid 2 \eta\right) e^{(\tau / 2) \partial_{z}}-\theta_{a+1}\left(-2 z-g+\frac{\tau}{2} \mid 2 \eta\right) e^{-(\tau / 2) \partial_{z}}\right] e^{-2 \pi i z^{2} / \tau},
\end{aligned}
$$

где $g$ - это тот же самый произвольный параметр, что и в (11). 
В рассматриваемой реализации операторы Казимира сводятся к скалярным выражениям

$$
\begin{array}{ll}
\mathbf{K}_{0}=4 \theta_{1}^{2}(g \mid \tau), & \mathbf{K}_{2}=4 \theta_{1}(g-\eta \mid \tau) \theta_{1}(g+\eta \mid \tau), \\
\widetilde{\mathbf{K}}_{0}=4 \theta_{1}^{2}(g \mid 2 \eta), & \widetilde{\mathbf{K}}_{2}=4 \theta_{1}\left(g-\frac{\tau}{2} \mid 2 \eta\right) \theta_{1}\left(g+\frac{\tau}{2} \mid 2 \eta\right),
\end{array}
$$

которые инвариантны относительно отражений $g \rightarrow-g$. Переменные $\eta$ и $\tau$ определяются структурными постоянными, поэтому спиновый параметр $g$ фиксирует значения всех операторов Казимира и характеризует представления эллиптического модулярного дубля. Существует другой эллиптический модулярный дубль [13], основанный на преобразовании $\eta \rightarrow 1 / 4 \eta, \tau \rightarrow \tau / 2 \eta, z \rightarrow z / 2 \eta, g \rightarrow g / 2 \eta$ в определении $(11)$, для которого допустим режим $\operatorname{Im} \eta=0$, но мы его здесь не рассматриваем.

\section{2. СПЛЕТАЮЩИЙ ОПЕРАТОР}

В настоящей статье мы фокусируем свое внимание на интегральном операторе, который был введен в работе [14] для определения универсального интегрального преобразования, задающего интегральный аналог техники цепочек Бейли [15]. Этот оператор действует на голоморфные функции $\Phi(x)$ одной комплексной переменной следующим образом:

$$
[\mathrm{M}(g) \Phi](z)=\frac{(q ; q)_{\infty}(p ; p)_{\infty}}{2} \int_{0}^{1} \frac{\Gamma( \pm z \pm x-g)}{\Gamma(-2 g, \pm 2 x)} \Phi(x) d x
$$

где $\operatorname{Im}(-g \pm z)>0$. Мы используем обозначения $\Gamma(a, b \pm z):=\Gamma(a) \Gamma(b+z) \Gamma(b-z)$, где

$$
\Gamma(z):=\Gamma(z \mid \tau, 2 \eta):=\prod_{n, m=0}^{\infty} \frac{1-e^{-2 \pi i z} p^{n+1} q^{m+1}}{1-e^{2 \pi i z} p^{n} q^{m}}
$$

есть эллиптическая гамма-функция, определенная при $|p|,|q|<1$. Ограничения для параметра $g$ и аргумента $z$ могут быть ослаблены деформацией контура интегрирования, т.е. путем аналитического продолжения приведенного выше выражения (в тех ситуациях, когда это разрешено функциями, на которые действует данный оператор).

Сплетающий оператор удовлетворяет очень простому соотношению отражения [16], напоминающему ключевое свойство преобразования Фурье:

$$
\mathrm{M}(g) \mathrm{M}(-g)=\mathbb{1} .
$$

Это равенство справедливо для подходящего пространства тестовых функций, по крайней мере для значений параметра $g$, которые не лежат на дискретных решетках $g=n \eta+m \tau / 2$ и $g=1 / 2+n \eta+m \tau / 2$, где $n, m \in \mathbb{Z}[12]$.

Как показано в работе [12], оператор (16), будучи симметричным по $2 \eta$ и $\tau$, удовлетворяет следующим сплетающим соотношениям:

$$
\mathrm{M}(g) \mathbf{S}^{a}(g)=\mathbf{S}^{a}(-g) \mathrm{M}(g), \quad \mathrm{M}(g) \widetilde{\mathbf{S}}^{a}(g)=\widetilde{\mathbf{S}}^{a}(-g) \mathrm{M}(g) .
$$

Здесь мы явно указываем зависимость от спина $g$ для генераторов алгебры Склянина, чтобы показать, что при действии оператора М параметр $g$ просто меняет знак. Поскольку это не меняет значений операторов Казимира, оператор $\mathrm{M}(g)$ связывает 
между собой эквивалентные представления. В общепринятом рассмотрении алгебры Склянина используется параметризация $g=\eta(2 \ell+1)$, для которой подразумевается преобразование $\ell \rightarrow-1-\ell$. Равенства (19) показывают, что ядро оператора М образует инвариантное пространство для эллиптического модулярного дубля, т. е. оно инвариантно относительно действия генераторов алгебры Склянина $\mathbf{S}^{a}(g)(11)$ и $\widetilde{\mathbf{S}}^{a}(g)(15)$.

Нетривиальное конечномерное ядро оператора М было построено в нашей предыдущей работе [12], здесь мы описываем это пространство более детальным образом. Сплетающие операторы являются очень важными инструментами в теории представлений [17], [18], в которой они используются для исследования вопросов редуцируемости, таких как существование и характеризация инвариантных подпространств для заданного бесконечномерного представления. Кроме того, в теории уравнения ЯБ [19] они могут служить элементарными строительными блоками для $R$-операторов.

Сплетающий оператор $\mathrm{M}(g)$ играет ключевую роль в построении наиболее сложного известного решения уравнения ЯБ [12], найденного в соответствии с общей конструкцией из работ [20], [21]. Этот подход к уравнению ЯБ основан на твистованном представлении генераторов группы перестановок. В нашем случае необходимые соотношения Кокстера удовлетворяются как следствие свойств эллиптического бетаинтеграла [22] и леммы Бейли из работы [14]. В настоящей статье мы не применяем полученные результаты к построению решений уравнения ЯБ, откладывая эту задачу для отдельной работы.

Введем обозначения

$$
Z=e^{2 \pi i z}, \quad X=e^{2 \pi i x}, \quad t=e^{-2 \pi i g}, \quad \Gamma_{p, q}(Z):=\Gamma(z \mid \tau, 2 \eta) .
$$

Предположим, что оператор $\mathrm{M}(g)$ действует в пространстве голоморфных функций переменной $X$. Тогда мы можем переписать сплетающий оператор в виде

$$
[\mathrm{M}(g) f](Z)=\frac{(q ; q)_{\infty}(p ; p)_{\infty}}{4 \pi i} \int_{\mathbb{T}} \frac{\Gamma_{p, q}\left(t Z^{ \pm 1} X^{ \pm 1}\right)}{\Gamma_{p, q}\left(t^{2}, X^{ \pm 2}\right)} f(X) \frac{d X}{X},
$$

где $\mathbb{T}$ - единичная окружность с положительной ориентацией, $\left|t Z^{ \pm 1}\right|<1$ и

$$
\Gamma_{p, q}\left(a, b^{ \pm k}\right):=\Gamma_{p, q}(a) \Gamma_{p, q}\left(b^{k}\right) \Gamma_{p, q}\left(b^{-k}\right) .
$$

Отметим, что требование голоморфности по переменной $X$ эквивалентно требованию периодичности функций $\Phi(x)$ в $(16), \Phi(x+1)=\Phi(x)$, что сильно ограничивает пространство используемых функций. Однако такое требование является естественным, так как после действия сплетающего оператора М всегда появляются мероморфные функции переменной $Z$.

\section{3. СООТНОШЕНИЯ СМЕЖНОСТИ ДЛЯ СПЛЕТАЮЩЕГО ОПЕРАТОРА}

Соотношения смежности (или рекуррентные соотношения) связывают друг с другом специальные функции с различными значениями параметров [15]. Первое соотношение смежности для эллиптических гипергеометрических интегралов было сконструировано еще в работе [22]. Такие соотношения можно написать также для интегральных операторов, и мы хотели бы сделать это для сплетающего оператора $\mathrm{M}(\mathrm{g})$. 
Интересующее нас рекуррентное соотношение для оператора (16) имеет следующий вид [23]:

$$
\begin{aligned}
& \frac{e^{\pi i \eta}}{\mathrm{R}(\tau)} \frac{e^{\pi i z^{2} / \eta}}{\theta_{1}(2 z)}\left[\bar{\theta}_{k}(z+g+\eta) e^{\eta \partial_{z}}-\bar{\theta}_{k}(z-g-\eta) e^{-\eta \partial_{z}}\right] e^{-\pi i z^{2} / \eta} \mathrm{M}(g)= \\
& \quad=\mathrm{M}(g+\eta) \bar{\theta}_{k}(z)
\end{aligned}
$$

где $\bar{\theta}_{k}(z)=\theta_{k}(z \mid \tau / 2), k=3,4$. Здесь $\mathrm{R}(\tau)$ - постоянная, определенная в (6). В правой части равенства (21) и в других выражениях, приведенных ниже, мы используем переменную $z$ и подразумеваем, что она является “внутренней” переменной, т. е. играет роль $x$ в определении интегрального оператора (16). Приведенное соотношение может быть записано как равенство

$$
\mathrm{A}_{k}(g) \mathrm{M}(g)=\mathrm{M}(g+\eta) \bar{\theta}_{k}(z),
$$

где $\mathrm{A}_{k}(g)$ обозначает следующий разностный оператор:

$$
\mathrm{A}_{k}(g)=\frac{e^{\pi i \eta}}{\mathrm{R}(\tau)} e^{\pi i z^{2} / \eta} \frac{1}{\theta_{1}(2 z)}\left[\bar{\theta}_{k}(z+g+\eta) e^{\eta \partial_{z}}-\bar{\theta}_{k}(z-g-\eta) e^{-\eta \partial_{z}}\right] e^{-\pi i z^{2} / \eta} .
$$

Для доказательства операторного тождества (21) мы отметим, что оно эквивалентно следующему уравнению для подынтегральной функции сплетающего оператора:

$$
\begin{gathered}
e^{\pi i z^{2} / \eta} \frac{1}{\theta_{1}(2 z)}\left[\bar{\theta}_{k}(z+g+\eta) e^{\eta \partial_{z}}-\bar{\theta}_{k}(z-g-\eta) e^{-\eta \partial_{z}}\right] e^{-\pi i z^{2} / \eta} \frac{\Gamma( \pm z \pm x-g)}{\Gamma(-2 g, \pm 2 x)}= \\
=\mathrm{R}(\tau) e^{-\pi i \eta} \frac{\Gamma( \pm z \pm x-g-\eta)}{\Gamma(-2 g-2 \eta, \pm 2 x)} \bar{\theta}_{k}(x) .
\end{gathered}
$$

Доказательство этого соотношения основывается на двух ключевых формулах:

$$
\begin{gathered}
\Gamma(z+2 \eta)=\mathrm{R}(\tau) e^{\pi i z} \theta_{1}(z) \Gamma(z) \\
2 \theta_{1}(x \pm y):=2 \theta_{1}(x+y) \theta_{1}(x-y)=\bar{\theta}_{4}(x) \bar{\theta}_{3}(y)-\bar{\theta}_{4}(y) \bar{\theta}_{3}(x) .
\end{gathered}
$$

Мы имеем равенства

$$
\begin{aligned}
& e^{\pi i z^{2} / \eta} e^{\eta \partial_{z}} e^{-\pi i z^{2} / \eta} \Gamma( \pm z \pm x-g)= \\
& \quad=\mathrm{R}^{2}(\tau) e^{-3 \pi i \eta-2 \pi i g} \theta_{1}(z-\eta-g \pm x) \Gamma( \pm z \pm x-g-\eta) \\
& e^{\pi i z^{2} / \eta} e^{-\eta \partial_{z}} e^{-\pi i z^{2} / \eta} \Gamma( \pm z \pm x-g)= \\
& \quad=\mathrm{R}^{2}(\tau) e^{-3 \pi i \eta-2 \pi i g} \theta_{1}(z+\eta+g \pm x) \Gamma( \pm z \pm x-g-\eta)
\end{aligned}
$$

поэтому

$$
\begin{aligned}
e^{\pi i z^{2} / \eta} \frac{1}{\theta_{1}(2 z)}[ & \left.\bar{\theta}_{k}(z+g+\eta) e^{\eta \partial_{z}}-\bar{\theta}_{k}(z-g-\eta) e^{-\eta \partial_{z}}\right] e^{-\pi i z^{2} / \eta} \Gamma( \pm z \pm x-g)= \\
= & \mathrm{R}^{2}(\tau) e^{-3 \pi i \eta-2 \pi i g} \cdot \frac{1}{\theta_{1}(2 z)} \Gamma( \pm z \pm x-g-\eta) \times \\
& \left.\times \bar{\theta}_{k}(z+g+\eta) \theta_{1}(z-\eta-g \pm x)-\bar{\theta}_{k}(z-g-\eta) \theta_{1}(z+\eta+g \pm x)\right]= \\
= & \mathrm{R}^{2}(\tau) e^{-3 \pi i \eta-2 \pi i g} \Gamma( \pm z \pm x-g-\eta) \theta_{1}(-2 g-2 \eta) \bar{\theta}_{k}(x) .
\end{aligned}
$$


В последней строке мы использовали равенство

$$
\begin{gathered}
\bar{\theta}_{k}(z+g+\eta) \theta_{1}(z-\eta-g \pm x)-\bar{\theta}_{k}(z-g-\eta) \theta_{1}(z+\eta+g \pm x)= \\
=\theta_{1}(2 z) \theta_{1}(-2 g-2 \eta) \bar{\theta}_{k}(x),
\end{gathered}
$$

которое получается двукратным применением соотношения (26). На заключительном шаге мы применяем равенство

$$
\frac{\theta_{1}(-2 g-2 \eta)}{\Gamma(-2 g)}=\frac{\mathrm{R}^{-1}(\tau) e^{2 \pi i(g+\eta)}}{\Gamma(-2 g-2 \eta)}
$$

и получаем тождество (24).

Предположим, что параметр $g$ удовлетворяет условиям, гарантирующим выполнение соотношения обращения (18). Тогда легко получить другое рекуррентное соотношение

$$
\bar{\theta}_{k}(z) \mathrm{M}(g+\eta)=\mathrm{M}(g) \frac{e^{\pi i \eta}}{\mathrm{R}(\tau)} \frac{e^{\pi i z^{2} / \eta}}{\theta_{1}(2 z)}\left[\bar{\theta}_{k}(z-g) e^{\eta \partial_{z}}-\bar{\theta}_{k}(z+g) e^{-\eta \partial_{z}}\right] e^{-\pi i z^{2} / \eta} .
$$

Действительно, умножая равенство (22) на оператор $\mathrm{M}^{-1}(g+\eta)$, мы получаем

$$
\mathrm{M}^{-1}(g+\eta) \mathrm{A}_{k}(g)=\bar{\theta}_{k}(z) \mathrm{M}^{-1}(g), \quad \text { или } \quad \mathrm{M}(-g-\eta) \mathrm{A}_{k}(g)=\bar{\theta}_{k}(z) \mathrm{M}(-g) .
$$

Изменяя знак $g$ и сдвигая величину $g$ на $\eta$, получаем

$$
\mathrm{M}(g) \mathrm{A}_{k}(-g-\eta)=\bar{\theta}_{k}(z) \mathrm{M}(g+\eta),
$$

что совпадает с равенством (28). Однако равенство (28) можно получить без использования соотношения обращения при некоторых достаточно слабых ограничениях на пространство используемых функций. Действительно, операторное равенство (29) при действии на функцию $\Phi(x)$ имеет явный вид

$$
\begin{gathered}
\int_{0}^{1} \frac{\Gamma( \pm z \pm x-g)}{\mathrm{R}(\tau) \theta_{1}(2 x) \Gamma( \pm 2 x)}\left(\bar{\theta}_{k}(x-g) e^{-2 \pi i x} \Phi(x+\eta)-\bar{\theta}_{k}(x+g) e^{2 \pi i x} \Phi(x-\eta)\right) d x= \\
=\frac{\Gamma(-2 g)}{\Gamma(-2 g-2 \eta)} \bar{\theta}_{k}(z) \int_{0}^{1} \frac{\Gamma( \pm z \pm x-g-\eta)}{\Gamma( \pm 2 x)} \Phi(x) d x .
\end{gathered}
$$

Предположим теперь, что функция $\Phi(x)$ является периодической, $\Phi(x+1)=\Phi(x)$, и что подынтегральное выражение в левой части соотношения (30) не имеет простых полюсов в параллелограмме $[-\eta, \eta, 1+\eta, 1-\eta]$. Последнее условие означает, что подынтегральные функции в левой части (30) должны быть мероморфными функциями переменной $X=e^{2 \pi i x}$ без простых полюсов в кольце $|q|^{1 / 2} \leqslant|X| \leqslant|q|^{-1 / 2}$ при интересующих нас значениях $Z$, включая множество $|Z|=1$, для которого определено последовательное действие сплетающих операторов. При этом ограничении мы можем заменить функции $\Phi(x \pm \eta)$ в вышеприведенном уравнении на $\Phi(x)$ после подходящих сдвигов аргументов в подынтегральных функциях и свести интегрирования к интегрированию по интервалу $[0,1]$. Поскольку $\Phi(x)$ является произвольной функцией, мы получаем следующее уравнение на подынтегральные выражения:

$$
\begin{aligned}
& \frac{\Gamma( \pm z \pm(x-\eta)-g, \pm 2 x)}{\Gamma( \pm z \pm x-g, \pm 2(x-\eta))} \frac{e^{2 \pi i(\eta-x)}}{\mathrm{R}(\tau) \theta_{1}(2 x-2 \eta)} \bar{\theta}_{k}(x-g-\eta)- \\
& \quad-\frac{\Gamma( \pm z \pm(x+\eta)-g, \pm 2 x)}{\Gamma( \pm z \pm x-g, \pm 2(x+\eta))} \frac{e^{2 \pi i(\eta+x)}}{\mathrm{R}(\tau) \theta_{1}(2 x+2 \eta)} \bar{\theta}_{k}(x+g+\eta)=\frac{\Gamma(-2 g)}{\Gamma(-2 g-2 \eta)} \bar{\theta}_{k}(z) .
\end{aligned}
$$


После соответствующего упрощения отношений эллиптических гамма-функций мы в точности получаем тождество (27). Таким образом, второе операторное соотношение смежности (29) справедливо при более слабых ограничениях, чем необходимо для выполнения соотношения обращения (18).

\section{4. СПЛЕТАЮЩИЙ ОПЕРАТОР ДЛЯ ДВУХИНДЕКСНЫХ ДИСКРЕТНЫХ РЕШЕТОК $g=n \eta+m \tau / 2$$$
\text { И } g=1 / 2+n \eta+m \tau / 2 \text { ПРИ } n, m \in \mathbb{Z}_{\geqslant 0}
$$

Соотношения смежности для сплетающего оператора приводят к специфическому факторизованному виду оператора $\mathrm{M}(g)$ в специальных целочисленных точках спиновых решеток $g=n \eta+m \tau / 2$ и $g=1 / 2+n \eta+m \tau / 2$, где $n, m \in \mathbb{Z}_{\geqslant 0}$.

Можно повторить приведенные выше рассмотрения с заменой $2 \eta \rightleftarrows \tau$ и получить два типа рекуррентных соотношений (при $k=3,4$ )

$$
\mathrm{A}_{k}(g) \mathrm{M}(g)=\mathrm{M}(g+\eta) \theta_{k}\left(z \mid \frac{\tau}{2}\right), \quad \mathrm{B}_{k}(g) \mathrm{M}(g)=\mathrm{M}\left(g+\frac{\tau}{2}\right) \theta_{k}(z \mid \eta),
$$

где $\mathrm{A}_{k}(g)$ и $\mathrm{B}_{k}(g)$ обозначают разностные операторы

$$
\begin{aligned}
& \mathrm{A}_{k}(g)=e^{\pi i z^{2} / \eta} \frac{c_{\mathrm{A}}}{\theta_{1}(2 z \mid \tau)}\left[\theta_{k}\left(z+g+\eta \mid \frac{\tau}{2}\right) e^{\eta \partial_{z}}-\theta_{k}\left(z-g-\eta \mid \frac{\tau}{2}\right) e^{-\eta \partial_{z}}\right] e^{-\pi i z^{2} / \eta}, \\
& \mathrm{B}_{k}(g)=e^{2 \pi i z^{2} / \tau} \frac{c_{\mathrm{B}}}{\theta_{1}(2 z \mid 2 \eta)}\left[\theta_{k}\left(z+g+\frac{\tau}{2} \mid \eta\right) e^{(\tau / 2) \partial_{z}}-\theta_{k}\left(z-g-\frac{\tau}{2} \mid \eta\right) e^{-(\tau / 2) \partial_{z}}\right] e^{-2 \pi i z^{2} / \tau}
\end{aligned}
$$

и

$$
c_{A}=\frac{e^{\pi i \eta}}{\mathrm{R}(\tau)}, \quad c_{B}=\frac{e^{\pi i \tau / 2}}{\mathrm{R}(2 \eta)} .
$$

Используя начальное условие $\mathrm{M}(0)=\mathbb{1}$, которое доказывается простым анализом вычетов [12], можно разрешить рекуррентные соотношения и получить

$$
\begin{aligned}
\mathrm{M}^{(k)}(n \eta) & =\mathrm{A}_{k}(n \eta-\eta) \ldots \mathrm{A}_{k}(\eta) \mathrm{A}_{k}(0) \cdot \theta_{k}^{-n}\left(z \mid \frac{\tau}{2}\right), \\
\mathrm{M}^{(k)}\left(m \frac{\tau}{2}\right) & =\mathrm{B}_{k}\left(m \frac{\tau}{2}-\frac{\tau}{2}\right) \ldots \mathrm{B}_{k}\left(\frac{\tau}{2}\right) \mathrm{B}_{k}(0) \cdot \theta_{k}^{-m}(z \mid \eta) .
\end{aligned}
$$

Хотя вид сплетающего оператора не должен зависеть от значения $k, \mathrm{M}^{(k)}(g) \equiv \mathrm{M}(g)$, мы ввели дополнительный верхний индекс $k$ для того, чтобы указать на потенциальную зависимость от него.

Полученные выражения представляют собой частные случаи более общего оператора $\mathrm{M}^{(k)}(n \eta+m \tau / 2)$. Однако они используются как строительные элементы для построения этого общего оператора. Сначала мы представим эти факторизованные операторы в виде конечных сумм с явными коэффициентами. Рассмотрим для иллюстрации два простых примера:

$$
\begin{aligned}
e^{-\pi i z^{2} / \eta} \mathrm{M}^{(k)}(\eta) e^{\pi i z^{2} / \eta} & =\frac{c_{A}}{\theta_{1}(2 z)}\left[\theta_{k}\left(z+\eta \mid \frac{\tau}{2}\right) e^{\eta \partial_{z}}-\theta_{k}\left(z-\eta \mid \frac{\tau}{2}\right) e^{-\eta \partial_{z}}\right] \theta_{k}^{-1}\left(z \mid \frac{\tau}{2}\right)= \\
& =\frac{c_{A}}{\theta_{1}(2 z)}\left[e^{\eta \partial_{z}}-e^{-\eta \partial_{z}}\right]
\end{aligned}
$$

И

$$
\begin{aligned}
e^{-\pi i z^{2} / \eta} \mathrm{M}^{(k)}(2 \eta) e^{\pi i z^{2} / \eta}= & \frac{c_{A}^{2}}{\theta_{1}(2 z)}\left[\theta_{k}\left(z+2 \eta \mid \frac{\tau}{2}\right) e^{\eta \partial_{z}}-\theta_{k}\left(z-2 \eta \mid \frac{\tau}{2}\right) e^{-\eta \partial_{z}}\right] \times \\
& \times \frac{1}{\theta_{1}(2 z)}\left[e^{\eta \partial_{z}}-e^{-\eta \partial_{z}}\right] \theta_{k}^{-1}\left(z \mid \frac{\tau}{2}\right)
\end{aligned}
$$


Разлагая последнее выражение, мы получаем сумму четырех конечноразностных операторов, которые могут быть преобразованы с помощью тождества (26) к виду

$$
\begin{aligned}
e^{-\pi i z^{2} / \eta} \mathrm{M}^{(k)}(2 \eta) e^{\pi i z^{2} / \eta}= & \frac{c_{A}^{2}}{\theta_{1}(2 z-2 \eta) \theta_{1}(2 z) \theta_{1}(2 z+2 \eta)} \times \\
& \times\left[\theta_{1}(2 z-2 \eta) e^{2 \eta \partial_{z}}-\frac{\theta_{1}(4 \eta)}{\theta_{1}(2 \eta)} \theta_{1}(2 z)+\theta_{1}(2 z+2 \eta) e^{-2 \eta \partial_{z}}\right] .
\end{aligned}
$$

В этих примерах можно опустить индекс $k$ в обозначении $\mathrm{M}^{(k)}(n \eta)$, поскольку результат не зависит от $k$, как и ожидалось.

Рассмотрим общий анзац для сплетающего оператора,

$$
e^{-\pi i z^{2} / \eta} \mathrm{M}(n \eta) e^{\pi i z^{2} / \eta}=\sum_{\ell=0}^{n}(-1)^{\ell} \alpha_{\ell}^{(n)}(z) e^{(n-2 \ell) \eta \partial_{z}},
$$

и подставим его в равенство $\mathrm{M}((n+1) \eta)=\mathrm{A}_{k}(n \eta) \mathrm{M}(n \eta) \theta_{k}^{-1}(z \mid \tau / 2)$. При $\ell=1, \ldots, n$ это приводит к рекуррентным соотношениям

$$
\alpha_{\ell}^{(n+1)}(z)=\frac{c_{A}}{\theta_{1}(2 z \mid \tau)} \frac{\theta_{k}\left(z+(n+1) \eta \mid \frac{\tau}{2}\right) \alpha_{\ell}^{(n)}(z+\eta)+\theta_{k}\left(z-(n+1) \eta \mid \frac{\tau}{2}\right) \alpha_{\ell-1}^{(n)}(z-\eta)}{\theta_{k}\left(z+(n+1-2 \ell) \eta \mid \frac{\tau}{2}\right)} .
$$

При $\ell=0$ и $\ell=n+1$ имеем

$$
\alpha_{0}^{(n+1)}(z)=\frac{c_{A}}{\theta_{1}(2 z \mid \tau)} \alpha_{0}^{(n)}(z+\eta), \quad \alpha_{n+1}^{(n+1)}(z)=\frac{c_{A}}{\theta_{1}(2 z \mid \tau)} \alpha_{n}^{(n)}(z-\eta) .
$$

Поскольку $\alpha_{0}^{(0)}(z)=1$, мы получаем

$$
\alpha_{0}^{(n)}(z)=\frac{c_{A}^{n}}{\prod_{k=0}^{n-1} \theta_{1}(2 z+2 \eta k \mid \tau)}, \quad \alpha_{n}^{(n)}(z)=\frac{c_{A}^{n}}{\prod_{k=0}^{n-1} \theta_{1}(2 z-2 \eta k \mid \tau)} .
$$

Эти граничные значения $\alpha_{\ell}^{(n)}(z)$ фиксируют решение рекуррентного соотношения (33), которое может быть найдено по индукции:

$$
\begin{gathered}
\alpha_{\ell}^{(n)}(z)=c_{A}^{n} \cdot\left[\begin{array}{l}
n \\
\ell
\end{array}\right]_{\tau, 2 \eta} \frac{\theta_{1}(2 z+2 \eta(n-2 \ell) \mid \tau)}{\prod_{j=0}^{n} \theta_{1}(2 z-2 \eta(\ell-j) \mid \tau)}, \\
{\left[\begin{array}{c}
n \\
\ell
\end{array}\right]_{\tau, 2 \eta}=\frac{\prod_{j=1}^{n} \theta_{1}(2 \eta j \mid \tau)}{\prod_{j=1}^{\ell} \theta_{1}(2 \eta j \mid \tau) \cdot \prod_{j=1}^{n-\ell} \theta_{1}(2 \eta j \mid \tau)} .}
\end{gathered}
$$

В выражении для $\alpha_{\ell}^{(n)}(z)$ мы выделили эллиптический биномиальный коэффициент $\left[\begin{array}{l}n \\ \ell\end{array}\right]_{\tau, 2 \eta}$, который не зависит от $z$, и явно выписали остальную зависящую от $z$ часть. Дискретный сплетающий оператор $\mathrm{M}(n \eta)$ впервые был найден в работе [24] в этой форме. Как мы видим, действительно, общий результат не зависит от индекса $k$, который присутствует в рекуррентном соотношении для $\alpha_{\ell}^{(n)}(z)$.

Аналогичное выражение для оператора $\mathrm{M}(m \tau / 2)$ получается простой перестановкой $c_{A} \rightleftarrows c_{B}, n \rightleftarrows m$ и $\tau \rightleftarrows 2 \eta$ :

$$
e^{-2 \pi i z^{2} / \tau} \mathrm{M}\left(m \frac{\tau}{2}\right) e^{2 \pi i z^{2} / \tau}=\sum_{\ell=0}^{m}(-1)^{\ell} \beta_{\ell}^{(m)}(z) e^{(m-2 \ell)(\tau / 2) \partial_{z}},
$$


где

$$
\begin{gathered}
\beta_{\ell}^{(m)}(z)=c_{B}^{m} \cdot\left[\begin{array}{c}
m \\
\ell
\end{array}\right]_{2 \eta, \tau} \frac{\theta_{1}(2 z+\tau(m-2 \ell) \mid 2 \eta)}{\prod_{j=0}^{m} \theta_{1}(2 z-\tau(\ell-j) \mid 2 \eta)}, \\
{\left[\begin{array}{c}
m \\
\ell
\end{array}\right]_{2 \eta, \tau}=\frac{\prod_{j=1}^{m} \theta_{1}(\tau j \mid 2 \eta)}{\prod_{j=1}^{\ell} \theta_{1}(\tau j \mid 2 \eta) \prod_{j=1}^{m-\ell} \theta_{1}(\tau j \mid 2 \eta)} .}
\end{gathered}
$$

Теперь мы опишем общий случай. Легко получить следующее представление:

$$
\begin{aligned}
\mathrm{M}^{(k)}\left(n \eta+m \frac{\tau}{2}\right)= & \mathrm{A}_{k}\left(n \eta-\eta+m \frac{\tau}{2}\right) \ldots \mathrm{A}_{k}\left(\eta+m \frac{\tau}{2}\right) \mathrm{A}_{k}\left(m \frac{\tau}{2}\right) \times \\
& \times \mathrm{B}_{k}\left(m \frac{\tau}{2}-\frac{\tau}{2}\right) \ldots \mathrm{B}_{k}\left(\frac{\tau}{2}\right) \mathrm{B}_{k}(0) \cdot \theta_{k}^{-m}(z \mid \eta) \theta_{k}^{-n}\left(z \mid \frac{\tau}{2}\right) .
\end{aligned}
$$

Конечно, имеется много эквивалентных способов представить $\mathrm{M}^{(k)}(n \eta+m \tau / 2)$ в виде произведения операторов $\mathrm{A}_{k}$ и $\mathrm{B}_{k}$, и мы описали только один из них.

Мы можем рассмотреть аналогичным образом решетку $g=1 / 2+n \eta+m \tau / 2$. Необходимо использовать тот факт, что $\mathrm{M}(1 / 2)=P$, где $P=e^{(1 / 2) \partial_{z}}$ обозначает оператор сдвига $z$ на $1 / 2$ [12]. Поскольку мы работаем в пространстве функций, мероморфных по переменной $w=e^{2 \pi i z}$, сдвиг на половину периода эквивалентен преобразованию четности, $P w=-w$. Повторив предыдущую процедуру еще раз, в данном случае мы получаем

$$
\begin{aligned}
\mathrm{M}^{(k)}(n \eta & \left.+m \frac{\tau}{2}+\frac{1}{2}\right)=\mathrm{A}_{k}\left(n \eta-\eta+m \frac{\tau}{2}+\frac{1}{2}\right) \ldots \mathrm{A}_{k}\left(\eta+m \frac{\tau}{2}+\frac{1}{2}\right) \mathrm{A}_{k}\left(m \frac{\tau}{2}+\frac{1}{2}\right) \times \\
& \times \mathrm{B}_{k}\left(m \frac{\tau}{2}-\frac{\tau}{2}+\frac{1}{2}\right) \ldots \mathrm{B}_{k}\left(\frac{\tau}{2}+\frac{1}{2}\right) \mathrm{B}_{k}\left(\frac{1}{2}\right) \theta_{k}^{-m}\left(z+\frac{1}{2} \mid \eta\right) \theta_{k}^{-n}\left(z+\frac{1}{2} \mid \frac{\tau}{2}\right) P .
\end{aligned}
$$

Поскольку $\theta_{3}(z \pm 1 / 2)=\theta_{4}(z)$, мы видим, что

$$
\mathrm{A}_{3,4}\left(g+\frac{\tau}{2}\right)=\mathrm{A}_{4,3}(g), \quad \mathrm{B}_{3,4}\left(g+\frac{\tau}{2}\right)=\mathrm{B}_{4,3}(g) .
$$

Поэтому сплетающий оператор для второй решетки получается из первого случая перестановкой $\theta_{3}$ и $\theta_{4}$ и умножением на $P$ справа:

$$
\mathrm{M}^{(3,4)}\left(\frac{1}{2}+n \eta+m \frac{\tau}{2}\right)=\mathrm{M}^{(4,3)}\left(n \eta+m \frac{\tau}{2}\right) P .
$$

\section{5. КОНЕЧНОМЕРНОЕ ИНВАРИАНТНОЕ ПРОСТРАНСТВО ЭЛЛИПТИЧЕСКОГО МОДУЛЯРНОГО ДУБЛЯ}

Неприводимое представление алгебры Склянина для $g=n \eta$ при (полу)целых спинах $\ell=(n-1) / 2, n \in \mathbb{Z}_{>0}$, является $n$-мерным и может быть реализовано в пространстве $\Theta_{2 n-2}^{+}(z \mid \tau)$, состоящем из четных тета-функций от $z$ порядка $2 n-2$ с квазипериодами 1 и $\tau$ [11]. Пусть

$$
\mathrm{W}(g)=e^{-\pi i z^{2} / \eta} \mathrm{M}(g) e^{\pi i z^{2} / \eta} .
$$

Из факторизованного представления оператора $\mathrm{W}(\eta n)$ видно, что его действие аннигилирует элементы пространства $\Theta_{2 n-2}^{+}(z \mid \tau)$. Продемонстрируем это с помощью рекуррентных соотношений.

Функция $\theta_{k}^{2}(z \mid \tau)$ для любых $k=1,2,3,4$ является четной тета-функцией второго порядка. Зафиксируем два индекса $k_{1}, k_{2}, k_{1} \neq k_{2}$, и положим $e_{1}(z):=\theta_{k_{1}}^{2}(z \mid \tau)$ и $e_{2}(z)=\theta_{k_{2}}^{2}(z \mid \tau)$. Все мономы этих строительных блоков $e_{1}^{k}(z) e_{2}^{N-k}(z), k=0, \ldots, N$, 
являются четными тета-функциями порядка $2 N$. Они линейно независимы, т. е. равенство $\sum_{k=0}^{N} c_{k} e_{1}^{k}(z) e_{2}^{N-k}(z)=0$ выполяется только при $c_{k}=0$ (для проверки необходимо последовательно класть $z$ равным корню уравнения $\theta_{k_{1}}(z \mid \tau)=0$ или $\theta_{k_{2}}(z \mid \tau)=0$ после взятия подходящего числа производных по $\left.z\right)$. Число таких мономов определяет размерность пространства четных тета-функций порядка $2 N$, поэтому данные мономы образуют базис пространства $\Theta_{2 N}^{+}(z \mid \tau)$.

Используя теперь формулы

$$
\begin{aligned}
& 2 \theta_{1}(x+y) \theta_{1}(x-y)=\bar{\theta}_{4}(x) \bar{\theta}_{3}(y)-\bar{\theta}_{4}(y) \bar{\theta}_{3}(x), \\
& 2 \theta_{2}(x+y) \theta_{2}(x-y)=\bar{\theta}_{3}(x) \bar{\theta}_{3}(y)-\bar{\theta}_{4}(y) \bar{\theta}_{4}(x), \\
& 2 \theta_{3}(x+y) \theta_{3}(x-y)=\bar{\theta}_{3}(x) \bar{\theta}_{3}(y)+\bar{\theta}_{4}(y) \bar{\theta}_{4}(x), \\
& 2 \theta_{4}(x+y) \theta_{4}(x-y)=\bar{\theta}_{4}(x) \bar{\theta}_{3}(y)+\bar{\theta}_{4}(y) \bar{\theta}_{3}(x),
\end{aligned}
$$

можно записать любые $\theta_{k}^{2}(z \mid \tau)$ как линейные комбинации $\bar{\theta}_{3}(z)$ и $\bar{\theta}_{4}(z)$. Поэтому набор $n$ функций

$$
\theta_{3}^{j}\left(z \mid \frac{\tau}{2}\right) \theta_{4}^{n-1-j}\left(z \mid \frac{\tau}{2}\right), \quad j=0,1, \ldots, n-1,
$$

образует базис пространства $\Theta_{2 n-2}^{+}(z \mid \tau)$. Заметим, что эти функции можно умножать на произвольную четную тета-функцию нулевого порядка $e^{\alpha z^{2}}, \alpha \in \mathbb{C}$, так как они будут по-прежнему четными тета-функциями с некоторыми экспоненциальными множителями квазипериодичности, возникающими при сдвигах $z$ на 1 и $\tau$. Значение параметра $\alpha$, определяющее конкретную форму инвариантного пространства тета-функций, зависит от реализации генераторов алгебры Склянина. Стандартная форма генераторов, предложенная в работе [11], соответствует выбору $\alpha=0$.

Применяя $n-1$ раз рекуррентное соотношение и принимая во внимание равенство

$$
\mathrm{W}(\eta) \cdot 1=\frac{c_{A}}{\theta_{1}(2 z)}\left[e^{\eta \partial_{z}}-e^{-\eta \partial_{z}}\right] \cdot 1=0
$$

(здесь 1 можно заменить на произвольную $2 \eta$-периодическую функцию), мы получаем для $n>0$

$$
\mathrm{W}(n \eta) \theta_{3}^{j}\left(z \mid \frac{\tau}{2}\right) \theta_{4}^{n-1-j}\left(z \mid \frac{\tau}{2}\right)=0 \text {. }
$$

Таким образом, нулевые моды оператора $\mathrm{M}(n \eta)$ при $n>0$ имеют вид

$$
\mathrm{M}(n \eta) \phi_{j}(z) \psi_{j}(z)=0, \quad \phi_{j}(z):=e^{\pi i z^{2} / \eta} \theta_{3}^{j}\left(z \mid \frac{\tau}{2}\right) \theta_{4}^{n-1-j}\left(z \mid \frac{\tau}{2}\right)
$$

где $j=0,1, \ldots, n-1$ и $\psi_{j}(z)$ обозначает произвольную периодическую функцию, $\psi_{j}(z+2 \eta)=\psi_{j}(z)$.

Обычный базис конечномерного представления алгебры Склянина при (полу)целых значениях спина $\ell$ не содержит экспоненциального множителя $e^{\pi i z^{2} / \eta}$. Этот множитель появился из-за нестандартного вида генераторов алгебры (11), которые мы используем, и он не меняет пространства $\Theta_{2 n-2}^{+}$, будучи четной тета-функцией нулевого порядка. Отметим, однако, что он неаналитичен по переменной $e^{2 \pi i z}$, и это контрастирует с аналитической структурой сплетающего оператора $\mathrm{M}(g)$. 
Рассмотрим инвариантность полученного пространства нулевых мод под действием генераторов эллиптического модулярного дубля. Для $\mathbf{S}^{a}(n \eta)$ имеем

$$
\begin{aligned}
& \mathbf{S}^{a}(n \eta) \phi_{j}(z) \psi_{j}(z)=\psi_{j}(z+\eta) e^{\pi i z^{2} / \eta} \frac{i^{\delta_{a, 2}} \theta_{a+1}(\eta \mid \tau)}{\theta_{1}(2 z \mid \tau)} \times \\
& \quad \times\left[\theta_{a+1}(2 z-n \eta+\eta \mid \tau) e^{\eta \partial_{z}}-\theta_{a+1}(-2 z-n \eta+\eta \mid \tau) e^{-\eta \partial_{z}}\right] \theta_{3}^{j}\left(z \mid \frac{\tau}{2}\right) \theta_{4}^{n-1-j}\left(z \mid \frac{\tau}{2}\right) .
\end{aligned}
$$

Легко проверить, что действие разностного оператора в правой части приводит к тета-функции порядка $2 n-2$ (для этого достаточно убедиться в голоморфности этой функции и двойной квазипериодичности). Поэтому при фиксированной функции $\psi_{j}(z)=\psi(x)$, удовлетворяющей условию половинной (анти)периодичности $\psi(z+\eta)= \pm \psi(z)$, мы получаем конечномерное инвариантное пространство для обычной алгебры Склянина, найденное в работе [11]. Однако это пространство не инвариантно относительно действия генераторов модулярной пары $\widetilde{\mathbf{S}}^{a}$. Действительно, имеем

$$
\widetilde{\mathbf{S}}^{a}(n \eta) \phi_{j}(z) \psi(z)=(-1)^{n-1-j} e^{\pi i\left(\tau^{2} / 4 \eta-n \tau / 2\right)} \phi_{j}(z) i^{\delta_{a, 2}} \theta_{a+1}\left(\frac{\tau}{2} \mid 2 \eta\right) D^{a} \psi(z),
$$

где

$$
D^{a}=f_{a}(z) e^{(\tau / 2) \partial_{z}}+f_{a}(-z) e^{-(\tau / 2) \partial_{z}}
$$

и

$$
f_{a}(z):=e^{\pi i(\tau / \eta-2 n) z} \frac{\theta_{a+1}\left(2 z-n \eta+\frac{\tau}{2} \mid 2 \eta\right)}{\theta_{1}(2 z \mid 2 \eta)} .
$$

Таким образом, мы получим инвариантное пространство, если $\psi(z)$ будет собственной функцией всех четырех операторов $D^{a}$ одновременно, $D^{a} \psi(z)=\lambda_{a} \psi(z)$, что невозможно. Действительно, $f_{0,3}(z+\eta)=f_{0,3}(z)$ и $f_{1,2}(z+\eta)=-f_{1,2}(z)$. Так как $\psi(z+\eta)= \pm \psi(z)$, получаем $\lambda_{1,2}=0$, откуда следует противоречивое утверждение

$$
\frac{\psi\left(z+\frac{\tau}{2}\right)}{\psi\left(z-\frac{\tau}{2}\right)}=-\frac{f_{2}(-z)}{f_{2}(z)}=-\frac{f_{1}(-z)}{f_{1}(z)} .
$$

Выберем теперь указанные выше $2 \eta$-периодические функции в виде

$$
\psi_{j}(z)=\frac{e^{-\pi i z^{2} / \eta} \psi(z)}{\theta\left(q^{-1 / 4} Z^{ \pm 1} ; q\right)}, \quad \theta\left(t Z^{ \pm 1} ; q\right):=\theta(t Z ; q) \theta\left(t Z^{-1} ; q\right),
$$

и получим

$$
\mathrm{M}(n \eta) \varphi_{j}^{(n)}(z) \psi(z)=0, \quad \varphi_{j}^{(n)}(z):=\frac{\theta_{3}^{j}\left(z \mid \frac{\tau}{2}\right) \theta_{4}^{n-1-j}\left(z \mid \frac{\tau}{2}\right)}{\theta\left(q^{-1 / 4} Z^{ \pm 1} ; q\right)}, \quad j=0, \ldots, n-1 .
$$

В первом случае нулевые моды были голоморфны по $z$, но они не были аналитическими функциями переменной $Z$. Функции (42) мероморфны по $Z$, т. е. они имеют качественно другую аналитическую природу. Требование аналитичности по $Z$ приводит к редукции калибровочной свободы: на произвольный множитель $\psi(z)=\psi(z+2 \eta)$ теперь появляется дополнительное ограничение $\psi(z+1)=\psi(z)$. Это означает, что $\varphi_{j}^{(n)}(z)$ можно умножить на эллиптическую функцию $\chi(Z)$ такую, что $\chi(q Z)=\chi(Z)$. 
Рассмотрим инвариантность полученного пространства нулевых мод. Имеем

$$
\begin{aligned}
& \mathbf{S}^{a}(n \eta) \varphi_{j}^{(n)}(z) \chi(Z)=-\frac{\chi\left(q^{1 / 2} Z\right)}{\theta\left(q^{-1 / 4} Z^{ \pm 1} ; q\right)} \frac{i^{\delta_{a, 2}} \theta_{a+1}(\eta \mid \tau)}{\theta_{1}(2 z \mid \tau)} \times \\
& \quad \times\left[\theta_{a+1}(2 z-n \eta+\eta \mid \tau) e^{\eta \partial_{z}}-\theta_{a+1}(-2 z-n \eta+\eta \mid \tau) e^{-\eta \partial_{z}}\right] \theta_{3}^{j}\left(z \mid \frac{\tau}{2}\right) \theta_{4}^{n-1-j}\left(z \mid \frac{\tau}{2}\right) .
\end{aligned}
$$

Действие разностного оператора в правой части последнего равенства не выводит из пространства $\Theta_{2 n-2}^{+}(z \mid \tau)$. Поэтому мы будем иметь инвариантное пространство, если $\chi\left(q^{1 / 2} Z\right)= \pm \chi(Z)$.

Действие операторов $\widetilde{\mathbf{S}}^{a}(n \eta)$ имеет вид

$$
\widetilde{\mathbf{S}}^{a}(n \eta) \varphi_{j}^{(n)}(z) \chi(Z)=(-1)^{n-1-j} e^{-\pi i n \tau / 2} \varphi_{j}^{(n)}(z) i^{\delta_{a, 2}} \theta_{a+1}\left(\frac{\tau}{2} \mid 2 \eta\right) D^{a} \chi(Z),
$$

где $D^{a}$ задано формулой (41) и в данном случае

$$
f_{a}(z):=e^{-2 \pi i n z} \frac{\theta_{a+1}\left(2 z-n \eta+\frac{\tau}{2} \mid 2 \eta\right) \theta\left(q^{-1 / 4} Z^{ \pm 1} ; q\right)}{\theta_{1}(2 z \mid 2 \eta) \theta\left(q^{-1 / 4}\left(p^{1 / 2} Z\right)^{ \pm 1} ; q\right)} .
$$

Как и выше, $f_{0,3}(z+\eta)=f_{0,3}(z)$ и $f_{1,2}(z+\eta)=-f_{1,2}(z)$. Соответственно не существует функции $\chi(Z)$, которая была бы одновременно собственной функцией четырех операторов $D^{a}$.

Итак, для значений спина $g=n \eta, g=1 / 2+n \eta, g=m \tau / 2, g=1 / 2+m \tau / 2$ мы получаем конечномерные инвариантные пространства только для какой-либо одной из алгебр Склянина, входящих в эллиптический модулярный дубль.

Рассмотрим теперь другие дискретные значения спина $g$. Используя соотношения (31), можно записать

$$
\mathrm{M}\left(n \eta+\frac{\tau}{2}\right)=\mathrm{B}_{k}(n \eta) \mathrm{M}(n \eta) \theta_{k}^{-1}(z \mid \eta) .
$$

Перенесем множитель $\theta_{k}^{-1}(z \mid \eta)$ влево, пользуясь его квазипериодичностью и явным выражением для $\mathrm{M}(n \eta)$, получим

$$
\mathrm{M}\left(n \eta+\frac{\tau}{2}\right)=\widetilde{\mathrm{B}}(n \eta) \mathrm{W}(n \eta),
$$

где

$$
\begin{aligned}
\widetilde{\mathrm{B}}(n \eta)= & e^{2 \pi i z^{2} / \tau} \frac{c_{B}(-1)^{n \delta_{k, 4}}}{\theta_{1}(2 z \mid 2 \eta)} \times \\
& \times\left[\frac{\theta_{k}\left(z+n \eta+\frac{\tau}{2} \mid \eta\right)}{\theta_{k}\left(z+\frac{\tau}{2} \mid \eta\right)} e^{(\tau / 2) \partial_{z}}-\frac{\theta_{k}\left(z-n \eta-\frac{\tau}{2} \mid \eta\right)}{\theta_{k}\left(z-\frac{\tau}{2} \mid \eta\right)} e^{-(\tau / 2) \partial_{z}}\right] e^{-2 \pi i z^{2} / \tau} .
\end{aligned}
$$

Легко видеть, что отношения тета-функций в операторе $\widetilde{\mathrm{B}}$ можно упростить и что В пропорционален оператору $e^{(\tau / 2) \partial_{z}}-e^{-(\tau / 2) \partial_{z}}$, обложенному экспоненциальными множителями. Таким образом,

$$
\mathrm{M}\left(n \eta+\frac{\tau}{2}\right) \theta_{3}^{j}\left(z \mid \frac{\tau}{2}\right) \theta_{4}^{n-1-j}\left(z \mid \frac{\tau}{2}\right)=0, \quad j=0,1, \ldots, n-1, \quad n>0,
$$

т. е. те же самые функции, что и раньше, но уже без дополнительного множителя (экспоненциального или тета-функционального) являются нулевыми модами более 
сложного оператора $\mathrm{M}(n \eta+\tau / 2)$. При $n=0$ имеем $\mathrm{W}(0)=\mathbb{1}$, поэтому нулевая мода оператора $\mathrm{M}(\tau / 2)$ определяется множителем $\widetilde{\mathrm{B}}(0)$, и, очевидно, требование аналитичности по $Z$ приводит к функции $\chi(Z) / \theta\left(p^{-1 / 4} Z^{ \pm 1} ; p\right), \chi(p Z)=\chi(Z)$.

Точно так же, переставляя параметры $\tau$ и $2 \eta$, находим

$$
\mathrm{M}\left(m \frac{\tau}{2}\right) e^{2 \pi i z^{2} / \tau} \theta_{3}^{\ell}(z \mid \eta) \theta_{4}^{m-1-\ell}(z \mid \eta) \psi(z)=0, \quad \psi\left(z+\frac{\tau}{2}\right)= \pm \psi(z),
$$

где $\ell=0,1, \ldots, m-1, m>0$, или

$$
\mathrm{M}\left(m \frac{\tau}{2}\right) \frac{\theta_{3}^{\ell}(z \mid \eta) \theta_{4}^{m-1-\ell}(z \mid \eta)}{\theta\left(p^{-1 / 4} Z^{ \pm 1} ; p\right)} \chi(Z)=0, \quad \chi\left(p^{1 / 2} Z\right)= \pm \chi(Z) .
$$

Для $\mathrm{M}(m \tau / 2+\eta)$ получаем

$$
\mathrm{M}\left(m \frac{\tau}{2}+\eta\right) \theta_{3}^{\ell}(z \mid \eta) \theta_{4}^{m-1-\ell}(z \mid \eta)=0, \quad \ell=0,1, \ldots, m-1, \quad m>0 .
$$

Как будет показано ниже, каждая из $\mathrm{nm}$ функций

$$
\varphi_{j, \ell}^{(n, m)}(z):=\theta_{3}^{j}\left(z \mid \frac{\tau}{2}\right) \theta_{4}^{n-1-j}\left(z \mid \frac{\tau}{2}\right) \theta_{3}^{\ell}(z \mid \eta) \theta_{4}^{m-1-\ell}(z \mid \eta), \quad n, m>0,
$$

аннигилируется оператором $\mathrm{M}(n \eta+m \tau / 2)$,

$$
\mathrm{M}\left(n \eta+m \frac{\tau}{2}\right) \varphi_{j, \ell}^{(n, m)}(z)=0, \quad n, m>0 .
$$

Мы можем умножить $\varphi_{j, \ell}^{(n, m)}(z)$ на произвольные периодические функции с периодом либо $2 \eta$, либо $\tau$, и все равно это будут нулевые моды оператора $\mathrm{M}(n \eta+m \tau / 2)$.

Аналогичные свойства имеются и у оператора $\mathrm{M}(1 / 2+n \eta+m \tau / 2)$, поскольку сдвиг $z$ на $1 / 2$ просто переставляет $\theta_{3}(z)$ и $\theta_{4}(z)$, т. е. мы имеем перестановку базиса:

$$
\mathrm{M}\left(\frac{1}{2}+n \eta+m \frac{\tau}{2}\right) \varphi_{n-1-j, m-1-\ell}^{(n, m)}(z)=0, \quad n, m>0 .
$$

Ядро сплетающего оператора образует инвариантное подпространство при действии генераторов алгебры Склянина. Однако мы имеем дело только с конечномерным подпространством этого ядра. Поэтому необходимо убедиться, что оно является инвариантным. Для этого подействуем на $\varphi_{j, \ell}^{(n, m)}(z)$ операторами $\mathbf{S}^{a}(n \eta+m \tau / 2)$. Так как функции $\theta_{3,4}(z \mid \eta)$ квазипериодичны при сдвигах $z$ на $\eta$, имеем

$$
\begin{aligned}
& \mathbf{S}^{a}\left(n \eta+m \frac{\tau}{2}\right) \varphi_{j, \ell}^{(n, m)}(z)=(-1)^{m-1-\ell} e^{-\pi i m \eta} \theta_{3}^{\ell}(z \mid \eta) \theta_{4}^{m-1-\ell}(z \mid \eta) \frac{i^{\delta_{a, 2}} \theta_{a+1}(\eta \mid \tau)}{\theta_{1}(2 z \mid \tau)} \times \\
& \quad \times\left[e^{-2 \pi i m z} \theta_{a+1}\left(2 z-n \eta-m \frac{\tau}{2}+\eta \mid \tau\right) e^{\eta \partial_{z}}-\right. \\
& \left.\quad-e^{2 \pi i m z} \theta_{a+1}\left(-2 z-n \eta-m \frac{\tau}{2}+\eta \mid \tau\right) e^{-\eta \partial_{z}}\right] \theta_{3}^{\ell}\left(z \mid \frac{\tau}{2}\right) \theta_{4}^{m-1-\ell}\left(z \mid \frac{\tau}{2}\right) .
\end{aligned}
$$

Предположим, что целое число $m$ четно. Тогда в аргументах тета-функций $\theta_{a+1}$ стоят сдвиги на целые значения $\tau$, и, воспользовавшись квазипериодичностью $\theta_{a+1}$, мы получаем

$$
\begin{aligned}
\mathbf{S}^{a}(n \eta & \left.+m \frac{\tau}{2}\right) \varphi_{j, \ell}^{(n, m)}(z)=\mu_{a} e^{-\pi i m n \eta-\pi i m^{2} \tau / 4} \theta_{3}^{\ell}(z \mid \eta) \theta_{4}^{m-1-\ell}(z \mid \eta) \frac{i^{\delta_{a, 2}} \theta_{a+1}(\eta \mid \tau)}{\theta_{1}(2 z \mid \tau)} \times \\
& \times\left[\theta_{a+1}(2 z-n \eta+\eta \mid \tau) e^{\eta \partial_{z}}-\theta_{a+1}(-2 z-n \eta+\eta \mid \tau) e^{-\eta \partial_{z}}\right] \theta_{3}^{j}\left(z \mid \frac{\tau}{2}\right) \theta_{4}^{n-1-j}\left(z \mid \frac{\tau}{2}\right),
\end{aligned}
$$


где $\mu_{a}=(-1)^{m / 2+\ell+1}$ для $a=0,3$ и $\mu_{a}=(-1)^{\ell+1}$ для $a=1,2$. Видно, что правая часть содержит действие стандартных генераторов алгебры Склянина с $g=$ $n \eta$ в пространстве $\Theta_{2 n-2}^{+}(z \mid \tau)$, и мы имеем инвариантность пространства функций $\varphi_{j, \ell}^{(n, m)}(z)$ под действием генераторов $\mathbf{S}^{a}(n \eta+m \tau / 2)$. Переставляя $\eta$ и $\tau / 2$, а также $n$ и $m$, получаем то же самое утверждение и для генераторов $\widetilde{\mathbf{S}}^{a}(n \eta+m \tau / 2)$.

Пусть теперь $m$ - нечетное целое число. Тогда ситуация более сложная. Используя квазипериодичность $\theta_{a}$-функций при сдвигах аргумента на $\tau(m+1) / 2$, получаем

$$
\begin{aligned}
& \mathbf{S}^{a}\left(n \eta+m \frac{\tau}{2}\right) \varphi_{j, \ell}^{(n, m)}(z)=\mu_{a} e^{-\pi i(m n+n-1) \eta+\pi i(m+1) \tau / 2-\pi i(m+1)^{2} \tau / 4} \times \\
& \times \theta_{3}^{\ell}(z \mid \eta) \theta_{4}^{m-1-\ell}(z \mid \eta) \frac{i^{\delta_{a, 2}} \theta_{a+1}(\eta \mid \tau)}{\theta_{1}(2 z \mid \tau)}\left[e^{2 \pi i z} \theta_{a+1}\left(2 z-n \eta+\eta+\frac{\tau}{2} \mid \tau\right) e^{\eta \partial_{z}}-\right. \\
& \left.\quad-e^{-2 \pi i z} \theta_{a+1}\left(-2 z-n \eta+\eta+\frac{\tau}{2} \mid \tau\right) e^{-\eta \partial_{z}}\right] \theta_{3}^{j}\left(z \mid \frac{\tau}{2}\right) \theta_{4}^{n-1-j}\left(z \mid \frac{\tau}{2}\right),
\end{aligned}
$$

где $\mu_{a}=(-1)^{(m+1) / 2+\ell}$ для $a=0,3$ и $\mu_{a}=(-1)^{\ell}$ для $a=1,2$. Поскольку

$$
e^{\pi i z} \theta_{1,2,3,4}\left(z+\frac{\tau}{2} \mid \tau\right) \propto \theta_{4,3,2,1}(z \mid \tau)
$$

с точностью до постоянных множителей в правой части возникает перестановка действия стандартных генераторов Склянина $\mathbf{S}^{0,1,2,3} \rightarrow \mathbf{S}^{3,2,1,0}$, и мы приходим к инвариантности пространства функций $\varphi_{j, \ell}^{(n, m)}(z)$ для нечетных $m$.

Таким образом, при $n, m>0$ мы описали конечномерное (конкретно, $n m$-мерное) инвариантное пространство для алгебры Склянина, которое было частично охарактеризовано в работе [12]. В нем также реализуется конечномерное представление для эллиптического модулярного дубля. При $m=0$ или $n=0$ возникает конечномерное ( $n$ - или $m$-мерное) представление только для одной из алгебр Склянина, входящей в этот дубль. Наблюдение о том, что алгебра Склянина имеет конечномерные представления не только для значений спина $g=n \eta$, но и для целочисленных решеток $n \eta+m \tau / 2$ и $1 / 2+n \eta+m \tau / 2$ с $n, m>0$, является более или менее очевидным следствием модулярного дублирования, предложенного в статье [13], поскольку существует инволюция, переставляющая подалгебры Склянина. Этот факт был замечен также в работе [25].

Отметим, что эллиптический модулярный дубль однозначно определяет построенное конечномерное пространство. Если мы умножим указанные выше функции на какую-либо периодическую функцию $\psi(z)$ с периодом $\eta$ или $\tau / 2$, то условие инвариантности относительно действия генераторов дубля и требование аналитичности по переменной $e^{2 \pi i z}$ приводят к ограничениям $\psi(z+\eta)=\psi(z+\tau / 2)=\psi(z+1)=\psi(z)$. Благодаря несоизмеримости $2 \eta, \tau$ и 1 получаем $\psi(z)=$ const.

\section{6. НЕФАКТОРИЗОВАННАЯ ФОРМА СПЛЕТАЮЩЕГО ОПЕРАТОРА}

Теперь мы собираемся преобразовать общее выражение (36) к "нормально упорядоченному" виду. С помощью соотношений (7) легко показать, что

$$
\mathrm{A}_{k}\left(g+m \frac{\tau}{2}\right)=(-1)^{m \delta_{k, 4}} e^{-\pi i \tau m^{2} / 2-2 \pi i m(g+\eta)+\pi i m \eta} e^{\pi i m z^{2} / \eta} \mathrm{A}_{k}(g) e^{-\pi i m z^{2} / \eta},
$$


и поэтому

$$
\begin{aligned}
& \mathrm{A}_{k}\left(n \eta-\eta+m \frac{\tau}{2}\right) \ldots \mathrm{A}_{k}\left(\eta+m \frac{\tau}{2}\right) \mathrm{A}_{k}\left(m \frac{\tau}{2}\right)= \\
& \quad=(-1)^{m n \delta_{k, 4}} e^{-\pi i(\tau / 2) m^{2} n-\pi i \eta n^{2} m} e^{\pi i m z^{2} / \eta} \mathrm{A}_{k}(n \eta-\eta) \ldots \mathrm{A}_{k}(\eta) \mathrm{A}_{k}(0) e^{-\pi i m z^{2} / \eta} .
\end{aligned}
$$

Теперь можно выделить в $\mathrm{M}(n \eta+m \tau / 2)$ операторы $\mathrm{M}(n \eta)$ и $\mathrm{M}(m \tau / 2)$,

$$
\begin{aligned}
\mathrm{M}\left(n \eta+m \frac{\tau}{2}\right)= & (-1)^{m n \delta_{k, 4}} e^{-\pi i(\tau / 2) m^{2} n-\pi i \eta n^{2} m} \times \\
& \times e^{\pi i m z^{2} / \eta} \mathrm{M}(n \eta) e^{-\pi i m z^{2} / \eta} \theta_{k}^{n}\left(z \mid \frac{\tau}{2}\right) \mathrm{M}\left(m \frac{\tau}{2}\right) \theta_{k}^{-n}\left(z \mid \frac{\tau}{2}\right) .
\end{aligned}
$$

Используя свойства квазипериодичности функций $\theta_{k}^{-n}(z \mid \tau / 2)$, можно показать, что

$$
\theta_{k}^{n}\left(z \mid \frac{\tau}{2}\right) \mathrm{M}\left(m \frac{\tau}{2}\right) \theta_{k}^{-n}\left(z \mid \frac{\tau}{2}\right)=(-1)^{m n \delta_{k, 4}} e^{-2 \pi i n z^{2} / \tau} \mathrm{M}\left(m \frac{\tau}{2}\right) e^{2 \pi i n z^{2} / \tau} .
$$

Подставляя это соотношение в предыдущее равенство, мы получаем

$$
\begin{aligned}
& \mathrm{M}\left(n \eta+m \frac{\tau}{2}\right)=e^{-\pi i(\tau / 2) m^{2} n-\pi i \eta n^{2} m} e^{\pi i m z^{2} / \eta} \mathrm{M}(n \eta) e^{-\pi i m z^{2} / \eta-2 \pi i n z^{2} / \tau} \mathrm{M}\left(m \frac{\tau}{2}\right) e^{2 \pi i n z^{2} / \tau}= \\
& \quad=e^{-\pi i(\tau / 2) m^{2} n-\pi i \eta n^{2} m} e^{2 \pi i n z^{2} / \tau} \mathrm{M}\left(m \frac{\tau}{2}\right) e^{-\pi i m z^{2} / \eta-2 \pi i n z^{2} / \tau} \mathrm{M}(n \eta) e^{\pi i m z^{2} / \eta},
\end{aligned}
$$

где второе соотношение получено из первого простой перестановкой $n$ и $\eta$ с $m$ и $\tau / 2$.

Теперь легко показать с помощью рекуррентных соотношений справедливость равенства (44). Для этого рассмотрим действие оператора $\mathrm{M}(m \tau / 2) e^{2 \pi i n z^{2} / \tau}$, входящего в качестве крайнего правого множителя в формулу (46) для $\mathrm{M}(n \eta+m \tau / 2)$, на $\varphi_{j, \ell}^{(n, m)}(z)$,

$$
\begin{aligned}
& \mathrm{M}\left(m \frac{\tau}{2}\right) e^{2 \pi i n z^{2} / \tau} \varphi_{j, \ell}^{(n, m)}(z)=\mathrm{B}_{3}\left((m-1) \frac{\tau}{2}\right) \ldots \mathrm{B}_{3}\left((m-\ell) \frac{\tau}{2}\right) \times \\
& \quad \times \mathrm{B}_{4}\left((m-\ell-1) \frac{\tau}{2}\right) \ldots \mathrm{B}_{4}\left(\frac{\tau}{2}\right) \mathrm{M}\left(\frac{\tau}{2}\right) e^{2 \pi i n z^{2} / \tau} \theta_{3}^{j}\left(z \mid \frac{\tau}{2}\right) \theta_{4}^{n-1-j}\left(z \mid \frac{\tau}{2}\right),
\end{aligned}
$$

где мы воспользовались вторым рекуррентным соотношением в (31). Учитывая явный вид оператора $\mathrm{M}(\tau / 2)$ в правой части, находим

$$
\begin{aligned}
& \mathrm{M}\left(\frac{\tau}{2}\right) e^{2 \pi i n z^{2} / \tau} \theta_{3}^{j}\left(z \mid \frac{\tau}{2}\right) \theta_{4}^{n-1-j}\left(z \mid \frac{\tau}{2}\right)= \\
& \quad=\frac{c_{B}}{\theta_{1}(2 z \mid 2 \eta)} e^{2 \pi i z^{2} / \tau}\left(e^{(\tau / 2) \partial_{z}}-e^{-(\tau / 2) \partial_{z}}\right) e^{2 \pi i(n-1) z^{2} / \tau} \theta_{3}^{j}\left(z \mid \frac{\tau}{2}\right) \theta_{4}^{n-1-j}\left(z \mid \frac{\tau}{2}\right)=0,
\end{aligned}
$$

т.е. $\varphi_{j, \ell}^{(n, m)}(z)$ действительно определяют нулевые моды оператора $\mathrm{M}(n \eta+m \tau / 2)$. Более того, мы можем умножать $\varphi_{j, \ell}^{(n, m)}(z)$ на произвольные периодические функции $\psi(z+2 \eta)=\psi(z)$ или $\psi(z+\tau)=\psi(z)$ и все равно получать нулевые моды оператора $\mathrm{M}(n \eta+m \tau / 2)$.

Рассмотрим влияние преобразований подобия для обоих операторов, входящих в (46) в нефакторизованном виде. Необходимые формулы имеют вид

$$
\begin{gathered}
e^{\pi i(m+1) z^{2} / \eta} e^{(n-2 k) \eta \partial_{z}} e^{-\pi i(m+1) z^{2} / \eta}= \\
=e^{-2 \pi i z(m+1)(n-2 k)} e^{-\pi i \eta(m+1)(n-2 k)^{2}} e^{(n-2 k) \eta \partial_{z}}, \\
e^{-2 \pi i(n-1) z^{2} / \tau} e^{(m-2 \ell)(\tau / 2) \partial_{z}} e^{2 \pi i(n-1) z^{2} / \tau}= \\
=e^{2 \pi i z(n-1)(m-2 \ell)} e^{\pi i(\tau / 2)(n-1)(m-2 \ell)^{2}} e^{(m-2 \ell)(\tau / 2) \partial_{z}} .
\end{gathered}
$$

2 Теоретическая и математическая физика, т. 183, № 2, 2015 г. 
Теперь мы можем записать

$$
\mathrm{M}\left(n \eta+m \frac{\tau}{2}\right)=e^{-\pi i(\tau / 2) m^{2} n-\pi i \eta n^{2} m} \overline{\mathrm{M}}(n \eta) \overline{\mathrm{M}}^{\prime}\left(m \frac{\tau}{2}\right),
$$

где преобразованные операторы имеют вид

$$
\begin{aligned}
\overline{\mathrm{M}}(n \eta) & =\sum_{j=0}^{n}(-1)^{j} \alpha_{j}^{(n)}(z) e^{-2 \pi i z(m+1)(n-2 j)} e^{-\pi i \eta(m+1)(n-2 j)^{2}} e^{(n-2 j) \eta \partial_{z}}, \\
\overline{\mathrm{M}}^{\prime}\left(m \frac{\tau}{2}\right) & =\sum_{\ell=0}^{m}(-1)^{\ell} \beta_{\ell}^{(m)}(z) e^{2 \pi i z(n-1)(m-2 \ell)} e^{\pi i(\tau / 2)(n-1)(m-2 \ell)^{2}} e^{(m-2 \ell)(\tau / 2) \partial_{z}} .
\end{aligned}
$$

Перенесем все операторы сдвига в правую сторону, получим

$$
\begin{aligned}
\mathrm{M}\left(n \eta+m \frac{\tau}{2}\right)= & e^{-\pi i(\tau / 2) m^{2} n-\pi i \eta n^{2} m} \sum_{k=0}^{n}(-1)^{k} \alpha_{k}^{(n)}(z) e^{-2 \pi i z(m+1)(n-2 k)} \times \\
& \times e^{-\pi i \eta(m+1)(n-2 k)^{2}} \sum_{\ell=0}^{m}(-1)^{\ell} \beta_{\ell}^{(m)}(z+\eta(n-2 k)) \times \\
& \times e^{2 \pi i(z+\eta(n-2 k))(n-1)(m-2 \ell)} e^{\pi i(\tau / 2)(n-1)(m-2 \ell)^{2}} e^{(n-2 k) \eta \partial_{z}} e^{(m-2 \ell)(\tau / 2) \partial_{z}} .
\end{aligned}
$$

Используя формулы (7) преобразования тета-функций и явное выражение для коэффициентов $\beta^{(m)}$, мы получаем

$$
\beta_{\ell}^{(m)}(z+\eta(n-2 k))=(-1)^{n m} e^{4 \pi i z m(n-2 k)} e^{2 \pi i \eta m(n-2 k)^{2}} e^{\pi i \tau(m-1)(m-2 \ell)(n-2 k)} \beta_{\ell}^{(m)}(z) .
$$

Собирая всё вместе, получаем наш оператор в нормально упорядоченном виде:

$$
\begin{aligned}
\mathrm{M}\left(n \eta+m \frac{\tau}{2}\right)= & (-1)^{n m} e^{-\pi i(\tau / 2) m^{2} n-\pi i \eta n^{2} m} \sum_{k=0}^{n}(-1)^{k} \alpha_{k}^{(n)}(z) \times \\
& \times \sum_{\ell=0}^{m}(-1)^{\ell} \beta_{\ell}^{(m)}(z) e^{(n-1)(m-2 \ell)[\pi i(\tau / 2)(m-2 \ell)+2 \pi i(z+(n-2 k) \eta)]} \times \\
& \times e^{(m-1)(n-2 k)[\pi i \eta(n-2 k)+2 \pi i(z+(m-2 \ell)) \tau / 2]} e^{[(n-2 k) \eta+(m-2 \ell) \tau / 2] \partial_{z}},
\end{aligned}
$$

где мы записали фазовые множители в форме, напоминающей законы преобразования тета-функций определенного уровня при сдвиге на период.

Для решетки $g=1 / 2+n \eta+m \tau / 2$ имеем

$$
\mathrm{M}\left(\frac{1}{2}+n \eta+m \frac{\tau}{2}\right)=\mathrm{M}\left(n \eta+m \frac{\tau}{2}\right) P
$$

поскольку в соотношении (38) операторы $\mathrm{M}^{(k)}$ не зависят от $k$. 


\section{7. ПОЛНАЯ ФАКТОРИЗАЦИЯ СПЛЕТАЮЩЕГО ОПЕРАТОРА НА ТЕТА-ФУНКЦИЯХ}

Мы собираемся подействовать полученными сплетающими операторами на функции со специальными свойствами преобразования при сдвигах на периоды $\tau$ и $2 \eta$. Рассмотрим произведение $F_{N}(z) G_{M}(z)$, в котором функции $F_{N}(z)$ и $G_{M}(z)$ преобразуются следующим образом (здесь $a, b \in \mathbb{Z}$ )

$$
F_{N}(z+\tau a)=e^{-2 N a(\pi i \tau a+2 \pi i z)} F_{N}(z), \quad G_{M}(z+2 \eta b)=e^{-4 M b(\pi i \eta b+\pi i z)} G_{M}(z) .
$$

Мы записываем все фазовые множители в виде, близком к фазам в (47). Для голоморфных функций функции $F_{N}(z)$ являются тета-функциями модулярного параметра $\tau$ и имеют порядок $2 N$, в то время как $G_{M}(z)$ являются тета-функциями модулярного параметра $2 \eta$ и имеют порядок $2 M$.

Рассмотрим параметры $\alpha=0, \pm 1$ и $\beta=0, \pm 1$ такие, что $n-\alpha$ и $m-\beta$ всегда будут четными целыми числами. Это означает, что $\alpha=0$, когда $n$ четно, и $\alpha= \pm 1$, когда $n$ нечетно. Аналогично, $\beta=0$, когда $m$ четно, и $\beta= \pm 1$, когда $m$ нечетно. Теперь можно выделить сдвиги на полные периоды и показать, что

$$
\begin{aligned}
F_{N}\left(z+\beta \frac{\tau}{2}+(n\right. & \left.-2 k) \eta+(m-\beta-2 \ell) \frac{\tau}{2}\right) G_{M}\left(z+\alpha \eta+(n-\alpha-2 k) \eta+(m-2 \ell) \frac{\tau}{2}\right)= \\
= & e^{-N(m-\beta-2 \ell)[\pi i(\tau / 2)(m-2 \ell)+2 \pi i(z+\beta \tau / 2+(n-2 k) \eta)]} \times \\
& \times e^{-M(n-\alpha-2 k)[\pi i \eta(n-\alpha-2 k)+2 \pi i(z+\alpha \eta+(m-2 \ell) \tau / 2)]} \times \\
& \times F_{N}\left(z+\beta \frac{\tau}{2}+(n-2 k) \eta\right) G_{M}\left(z+\alpha \eta+(m-2 \ell) \frac{\tau}{2}\right) .
\end{aligned}
$$

Выбирая $N=n-1$ и $M=m-1$, мы наблюдаем почти полное сокращение фазовых множителей:

$$
\begin{aligned}
\mathrm{M}(n \eta+ & \left.m \frac{\tau}{2}\right) \\
= & F_{n-1}(z) G_{m-1}(z)= \\
& \times\left[\sum_{k=0}^{n m}(-1)^{k} \alpha_{k}^{(n)}(z) e^{-\pi i(\tau / 2) m^{2} n-\pi i \eta n^{2} m} e^{\pi i(\tau / 2) \beta^{2}(n-1)} e^{2 \pi i \eta \beta(n-1) n} e^{2 \pi i \beta(n-1) z} F_{n-1}\left(z+\beta \frac{\tau}{2}+(n-2 k) \eta\right)\right] \times \\
& \times e^{\pi i \eta \alpha^{2}(m-1)} e^{\pi i \tau \alpha(m-1) m} e^{2 \pi i \alpha(m-1) z} \times \\
& \times\left[\sum_{\ell=0}^{m}(-1)^{\ell} \beta_{\ell}^{(m)}(z) e^{-2 \pi i \tau \alpha(m-1) \ell} G_{m-1}\left(z+\alpha \eta+(m-2 \ell) \frac{\tau}{2}\right)\right] .
\end{aligned}
$$

Выбор $\alpha=\beta=0$ соответствует четным $n$ и $m$, и в этом случае все фазовые множители отсутствуют.

Таким образом, мы видим полную факторизацию сплетающего оператора - представление его в виде произведения двух операторов, действующих в различных пространствах. Заметим, что мы описали действие оператора М на произвольные тетафункции; при этом, как показано выше, нулевые моды оператора $\mathrm{M}(n \eta+m \tau / 2)$ выделяют пространства $\Theta_{2 n-2}^{+}(z \mid \tau)$ для $F_{n-1}$ и $\Theta_{2 m-2}^{+}(z \mid 2 \eta)$ для $G_{m-1}$.

Перейдем к мультипликативным обозначениям, которые более компактны и удобны по аналитическим причинам [12]. Напомним сначала, что

$$
p=e^{2 \pi i \tau}, \quad q=e^{4 \pi i \eta}, \quad \theta_{1}(z \mid \tau)=\frac{e^{-\pi i z} \theta\left(e^{2 \pi i z} ; p\right)}{\mathrm{R}(\tau)} .
$$


Подставляя последнее соотношение в определение эллиптических биномиальных коэффициентов, мы видим, что все коэффициенты $\mathrm{R}(\tau)$ сокращаются. Упрощая получающееся выражение с помощью равенства $\theta(z ; p)=-z \theta\left(z^{-1} ; p\right)$, мы получаем

$$
\left[\begin{array}{l}
n \\
k
\end{array}\right]_{\tau, 2 \eta}=(-1)^{k} q^{k(n+1) / 2} \prod_{b=1}^{k} \frac{\theta\left(q^{b-n-1} ; p\right)}{\theta\left(q^{b} ; p\right)}
$$

В оставшейся части коэффициентов $\alpha_{k}^{(n)}(z)$ множители $\mathrm{R}(\tau)$ также сокращаются, и мы находим, что

$$
c_{A}^{n} \frac{\theta_{1}(2 z+2 \eta(n-2 k) \mid \tau)}{\prod_{j=0}^{n} \theta_{1}(2 z-2 \eta(k-j) \mid \tau)}=q^{n / 4} q^{(n-2 k)(n-1) / 4} e^{2 \pi i n z} \frac{\theta\left(e^{4 \pi i z} q^{j-k} ; p\right)}{\prod_{j=0}^{n} \theta\left(e^{4 \pi i z} q^{j-k} ; p\right)} .
$$

Преобразуя тета-функции к подходящему виду и используя предыдущее выражение для эллиптических биномиальных коэффициентов, мы получаем

$$
\begin{aligned}
\alpha_{k}^{(n)}(z)= & \frac{(-1)^{k+1} q^{n^{2} / 4+n(k+1)} e^{2 \pi i(n+2) z}}{\prod_{j=0}^{n} \theta\left(e^{4 \pi i z} q^{j} ; p\right)} \times \\
& \times \theta\left(e^{-4 \pi i z} q^{2 k-n} ; p\right) \prod_{b=1}^{k} \frac{\theta\left(e^{-4 \pi i z} q^{b-n-1}, q^{b-n-1} ; p\right)}{\theta\left(e^{-4 \pi i z} q^{b}, q^{b} ; p\right)}
\end{aligned}
$$

Наконец, мы приводим явный вид сплетающего оператора в мультипликативных обозначениях:

$$
\begin{aligned}
\mathrm{M}\left(n \eta+m \frac{\tau}{2}\right) & F_{n-1}(z) G_{m-1}(z)=(-1)^{n m} e^{2 \pi i z[n+m+4+\alpha(m-1)+\beta(n-1)]} \times \\
& \times \frac{q^{\left(n^{2}-\alpha^{2}\right)(1-m) / 4+\beta n(n-1) / 2+n}}{\prod_{j=0}^{n} \theta\left(e^{4 \pi i z} q^{j} ; p\right)} \frac{p^{\left(m^{2}-\beta^{2}\right)(1-n) / 4+\alpha m(m-1) / 2+m}}{\prod_{j=0}^{m} \theta\left(e^{4 \pi i z} p^{j} ; q\right)} \times \\
& \times\left[\sum_{k=0}^{n} q^{k n(1-\beta)+\beta k} \theta\left(e^{-4 \pi i z} q^{2 k-n} ; p\right) \prod_{b=1}^{k} \frac{\theta\left(e^{-4 \pi i z} q^{b-n-1}, q^{b-n-1} ; p\right)}{\theta\left(e^{-4 \pi i z} q^{b}, q^{b} ; p\right)} \times\right. \\
& \left.\times F_{n-1}\left(z+\beta \frac{\tau}{2}+(n-2 k) \eta\right)\right] \times \\
\times & {\left[\sum_{\ell=0}^{m} p^{\ell m(1-\alpha)+\alpha \ell} \theta\left(e^{-4 \pi i z} p^{2 \ell-m} ; q\right) \prod_{b=1}^{\ell} \frac{\theta\left(e^{-4 \pi i z} p^{b-m-1}, p^{b-m-1} ; q\right)}{\theta\left(e^{-4 \pi i z} p^{b}, p^{b} ; q\right)} \times\right.} \\
& \left.\times G_{m-1}\left(z+\alpha \eta+(m-2 \ell) \frac{\tau}{2}\right)\right] .
\end{aligned}
$$

Выбирая в этой формуле $\alpha=\beta=1$, мы приходим к сплетающему оператору, полученному в работе [12] с помощью анализа вычетов (после изменения обозначений $w=e^{-2 \pi i z}, n=2 \ell_{q}+1$ и $\left.m=2 \ell_{p}+1\right):$

$$
\begin{aligned}
& \mathrm{M}\left(n \eta+m \frac{\tau}{2}\right) F_{n-1}(z) G_{m-1}(z)= \\
& \quad=\frac{(-1)^{n m} e^{4 \pi i z(n+m+1)} q^{\left(n^{2}-1\right)(1-m) / 4+(n+1) n / 2} p^{\left(m^{2}-1\right)(1-n) / 4+m(m+1) / 2}}{\prod_{j=0}^{n} \theta\left(e^{4 \pi i z} q^{j} ; p\right) \prod_{j=0}^{m} \theta\left(e^{4 \pi i z} p^{j} ; q\right)} \times
\end{aligned}
$$




$$
\begin{aligned}
& \times\left[\sum_{k=0}^{n} q^{k} \theta\left(e^{-4 \pi i z} q^{2 k-n} ; p\right) \prod_{b=1}^{k} \frac{\theta\left(e^{-4 \pi i z} q^{b-n-1}, q^{b-n-1} ; p\right)}{\theta\left(e^{-4 \pi i z} q^{b}, q^{b} ; p\right)} F_{n-1}\left(z+\frac{\tau}{2}+(n-2 k) \eta\right)\right] \times \\
& \times\left[\sum_{\ell=0}^{m} p^{\ell} \theta\left(e^{-4 \pi i z} p^{2 \ell-m} ; q\right) \prod_{b=1}^{\ell} \frac{\theta\left(e^{-4 \pi i z} p^{b-m-1}, p^{b-m-1} ; q\right)}{\theta\left(e^{-4 \pi i z} p^{b}, p^{b} ; q\right)} G_{m-1}\left(z+\eta+(m-2 \ell) \frac{\tau}{2}\right)\right] .
\end{aligned}
$$

Как показано выше, $\mathrm{M}(1 / 2+n \eta+m \tau / 2)=\mathrm{M}(n \eta+m \tau / 2) P$, поэтому для этого случая действие сплетающего оператора имеет тот же вид, что и ранее, со сдвигом $z$ в аргументах функций $F_{n-1}$ и $G_{m-1}$ на $1 / 2$.

\section{8. СПЛЕТАЮЩИЙ ОПЕРАТОР НА ДУАЛЬНОЙ РЕШЕТКЕ}

Рассмотрим более детально, что происходит с соотношением обращения (18) для сплетающего оператора, когда мы приближаемся к точкам решеток $g=n \eta+m \tau / 2$ и $g=1 / 2+n \eta+m \tau / 2, n, m \in \mathbb{Z}$. Сплетающий оператор (16) не определен для $g=-n \eta-m \tau / 2$ и $g=1 / 2-n \eta-m \tau / 2$, где $n, m \in \mathbb{Z}_{\geqslant 0}$ с исключением точек $(n, m)=(0,0),(0,1),(1,0)$. Действительно, множитель $1 / \Gamma(-2 g)$, входящий в него, имеет полюсы в точках этих решеток, в то время как часть, описывающаяся интегральным оператором, остается корректно определенной, поскольку $|t|<1$, $t=e^{-2 \pi i g}$. Поэтому удобно ввести перенормированный сплетающий оператор

$$
\left[\mathrm{M}_{\mathrm{ren}}(g) \Phi\right](z)=\frac{(q ; q)_{\infty}(p ; p)_{\infty}}{2} \int_{0}^{1} \frac{\Gamma( \pm z \pm x-g)}{\Gamma( \pm 2 x)} \Phi(x) d x
$$

Очевидно, что по-прежнему верны сплетающие соотношения

$$
\mathrm{M}_{\mathrm{ren}}(g) \mathbf{S}^{a}(g)=\mathbf{S}^{a}(-g) \mathrm{M}_{\mathrm{ren}}(g), \quad \mathrm{M}_{\mathrm{ren}}(g) \widetilde{\mathbf{S}}^{a}(g)=\widetilde{\mathbf{S}}^{a}(-g) \mathrm{M}_{\mathrm{ren}}(g) .
$$

Соотношения смежности (22) теперь принимают вид

$$
\begin{aligned}
& \mathrm{A}_{k}(g) \mathrm{M}_{\mathrm{ren}}(g)=\theta\left(e^{-4 \pi i(g+\eta)} ; p\right) \mathrm{M}_{\mathrm{ren}}(g+\eta) \theta_{k}\left(z \mid \frac{\tau}{2}\right), \\
& \mathrm{B}_{k}(g) \mathrm{M}_{\mathrm{ren}}(g)=\theta\left(e^{-4 \pi i(g+\tau / 2)} ; q\right) \mathrm{M}_{\mathrm{ren}}\left(g+\frac{\tau}{2}\right) \theta_{k}(z \mid \eta),
\end{aligned}
$$

где операторы $\mathrm{A}_{k}(g)$ и $\mathrm{B}_{k}(g)$ имеют тот же вид, что и раньше. Можно модифицировать аналогичным образом и сопутствующие соотношения (29).

Легко получить явное выражение для сплетающего оператора $\mathrm{M}_{\mathrm{ren}}(-n \eta-m \tau / 2)$, $n, m \in \mathbb{Z}_{\geqslant 0},(n, m) \neq(0,0)$. В мультипликативных обозначениях имеем

$$
\begin{aligned}
{\left[\mathrm{M}_{\mathrm{ren}}(-n \eta\right.} & \left.\left.-m \frac{\tau}{2}\right) \Phi\right](Z)=\frac{(p ; p)_{\infty}(q ; q)_{\infty}}{4 \pi i} \int_{\mathbb{T}} \frac{\Phi(X)}{\Gamma_{p, q}\left(X^{ \pm 2}\right)} \frac{d X}{X} \times \\
& \times \frac{\prod_{i=0}^{n-1} \theta\left(q^{i-n / 2} p^{m / 2} X Z^{ \pm 1} ; p\right) \prod_{k=0}^{m-1} \theta\left(q^{-n / 2} p^{k-m / 2} X Z^{ \pm 1} ; q\right)}{\theta\left(q^{n / 2} p^{m / 2} X^{-1} Z^{ \pm 1} ; p\right) \theta\left(q^{-n / 2} p^{-m / 2} X Z^{ \pm 1} ; q\right)}
\end{aligned}
$$

Рассмотрим теперь рекуррентное соотношение (22) при $g=-\eta$,

$$
\mathrm{A}_{k}(-\eta) \mathrm{M}(-\eta)=\mathrm{M}(0) \bar{\theta}_{k}(z)=\bar{\theta}_{k}(z)
$$


где

$$
\mathrm{A}_{k}(-\eta)=e^{\pi i z^{2} / \eta} \frac{c_{A} \theta_{k}\left(z \mid \frac{\tau}{2}\right)}{\theta_{1}(2 z \mid \tau)}\left[e^{\eta \partial_{z}}-e^{-\eta \partial_{z}}\right] e^{-\pi i z^{2} / \eta} .
$$

Таким образом, мы получаем операторное тождество

$$
\frac{c_{A} e^{-\pi i \eta}}{\theta_{1}(2 z \mid \tau)}\left[e^{-2 \pi i z} e^{\eta \partial_{z}}-e^{2 \pi i z} e^{-\eta \partial_{z}}\right] \mathrm{M}(-\eta)=\mathbb{1}, \quad \text { или } \quad \mathrm{M}(\eta) \mathrm{M}(-\eta)=\mathbb{1},
$$

которое следует из представления для $\mathrm{M}(\eta)$ в формуле (32). Это соотношение явно запрещает оператору $\mathrm{M}(-\eta)$ иметь нулевые моды. Заметим, что если сделать заключение о справедливости равенства $\mathrm{M}(-\eta) \mathrm{M}(\eta)=\mathbb{1}$ в качестве подобного следствия из дуального соотношения смежности (29), то это не будет верно. Как показано выше, оператор $\mathrm{M}(-\eta)$ имеет сингулярную подынтегральную функцию $1 / \theta\left(e^{2 \pi i(x-\eta \pm z)} ; q\right)$, и при сдвигах $z \rightarrow z \pm \eta$, использовавшихся при выводе (29), сингулярности типа полюсов садятся на контур интегрирования, при этом рекуррентное соотношение не выполняется. И, действительно, соотношение $\mathrm{M}(-\eta) \mathrm{M}(\eta)=\mathbb{1}$ не может быть верным, так как мы знаем, что оператор $\mathrm{M}(\eta)$ обладает нетривиальными нулевыми модами. Аналогичным образом мы получаем равенства

$$
\mathrm{M}\left(\frac{1}{2}+\eta\right) \mathrm{M}\left(\frac{1}{2}-\eta\right)=\mathrm{M}\left(\frac{\tau}{2}\right) \mathrm{M}\left(-\frac{\tau}{2}\right)=\mathrm{M}\left(\frac{1+\tau}{2}\right) \mathrm{M}\left(\frac{1-\tau}{2}\right)=\mathbb{1}
$$

без простых соотношений для $\mathrm{M}(-\tau / 2) \mathrm{M}(\tau / 2)$ и $\mathrm{M}((1-\tau) / 2) \mathrm{M}((1+\tau) / 2)$.

Возьмем теперь соотношение обращения (18), умножим его на множитель $\Gamma(-2 g)$ и рассмотрим предел $g \rightarrow n \eta+m \tau / 2$ или $g \rightarrow 1 / 2+n \eta+m \tau / 2$, где $n, m \in \mathbb{Z}_{\geqslant 0}$. Как упоминалось выше, в этом пределе функция $\Gamma(-2 g)$ обращается в ноль, за исключением точек $(n, m)=(0,0),(1,0),(0,1)$, для которых оператор (53) имеет сингулярное подынтегральное выражение. Исключая их, мы формально приходим к операторным равенствам

$$
\mathrm{M}\left(n \eta+m \frac{\tau}{2}\right) \mathrm{M}_{\mathrm{ren}}\left(-n \eta-m \frac{\tau}{2}\right)=\mathrm{M}\left(\frac{1}{2}+n \eta+m \frac{\tau}{2}\right) \mathrm{M}_{\mathrm{ren}}\left(\frac{1}{2}-n \eta-m \frac{\tau}{2}\right)=0 .
$$

Аналогично мы можем умножить (18) на множитель $\Gamma(2 g)$ и рассмотреть предел $g \rightarrow-n \eta-m \tau / 2$ или $g \rightarrow 1 / 2-n \eta-m \tau / 2$, где $n, m \in \mathbb{Z}_{\geqslant 0}$. Опять, за исключением значений $(n, m)=(0,0),(1,0),(0,1)$, мы получаем

$$
\mathrm{M}_{\text {ren }}\left(-n \eta-m \frac{\tau}{2}\right) \mathrm{M}\left(n \eta+m \frac{\tau}{2}\right)=\mathrm{M}_{\text {ren }}\left(\frac{1}{2}-n \eta-m \frac{\tau}{2}\right) \mathrm{M}\left(\frac{1}{2}+n \eta+m \frac{\tau}{2}\right)=0 .
$$

Докажем равенства (55), используя полученные ранее результаты. Рассмотрим часть подынтегральной функции оператора (53), зависящую от "внешней" переменной $Z$. Пусть $n, m>0$. Тогда тета-функции в знаменателе сокращаются, и с помощью первого соотношения в (39) произведения тета-функций в числителе можно представить в виде линейной комбинации функций $\varphi_{j, \ell}^{(n, m)}(z)$, заданных в (43). Как уже было показано ранее, эти функции являются нулевыми модами оператора $\mathrm{M}(n \eta+m \tau / 2)$ при $n, m>0$.

Положим теперь $m=0$. Рассмотрим действие оператора $\mathrm{M}(n \eta)$ на часть подынтегральной функции $\mathrm{M}_{\mathrm{ren}}(-n \eta)$, зависящей от $Z$ :

$$
\mathrm{M}(n \eta) \frac{\prod_{i=0}^{n-1} \theta\left(q^{i-n / 2} X Z^{ \pm 1} ; p\right)}{\theta\left(q^{n / 2} X^{-1} Z^{ \pm 1} ; p\right) \theta\left(q^{-n / 2} X Z^{ \pm 1} ; q\right)} .
$$


Поскольку $\theta\left(q^{n / 2} X^{-1} Z^{ \pm 1} ; p\right)=q^{n} X^{-2} \theta\left(q^{-n / 2} X Z^{ \pm 1} ; p\right)$, мы можем сократить часть тета-функций. В результате в числителе остается произведение

$$
\prod_{i=1}^{n-1} \theta\left(q^{i-n / 2} X Z^{ \pm 1} ; p\right)
$$

которое можно представить в виде линейной комбинации тета-функций

$$
\theta_{3}^{j}\left(z \mid \frac{\tau}{2}\right) \theta_{4}^{n-1-j}\left(z \mid \frac{\tau}{2}\right), \quad j=0, \ldots, n-1 .
$$

Следовательно, нам необходимо доказать, что

$$
\mathrm{M}(n \eta) \frac{\theta_{3}^{j}\left(z \mid \frac{\tau}{2}\right) \theta_{4}^{n-1-j}\left(z \mid \frac{\tau}{2}\right)}{\theta\left(q^{-n / 2} X Z^{ \pm 1} ; q\right)}=0 .
$$

Действительно, применяя рекуррентное соотношение (22), находим

$$
\begin{aligned}
\mathrm{M}(n \eta) & \frac{\theta_{3}^{j}\left(z \mid \frac{\tau}{2}\right) \theta_{4}^{n-1-j}\left(z \mid \frac{\tau}{2}\right)}{\theta\left(X Z^{ \pm 1} ; q\right)}= \\
& =\mathrm{A}_{3}((n-1) \eta) \ldots \mathrm{A}_{3}((n-j) \eta) \mathrm{A}_{4}((n-j-1) \eta) \ldots \mathrm{A}_{4}(\eta) \mathrm{M}(\eta) \frac{1}{\theta\left(X Z^{ \pm 1} ; q\right)}
\end{aligned}
$$

Пользуясь явным видом оператора $\mathrm{M}(\eta)$, получаем

$$
\begin{aligned}
\mathrm{M}(\eta) \frac{1}{\theta\left(X Z^{ \pm 1} ; q\right)}= & \frac{1}{\theta\left(X\left(q^{1 / 2} Z\right)^{ \pm 1} ; q\right)} \frac{c_{A} e^{\pi i z^{2} / \eta}}{\theta_{1}(2 z \mid \tau)} \times \\
& \times\left(e^{\eta \partial_{z}}-\frac{\theta\left(X\left(q^{1 / 2} Z\right)^{ \pm 1} ; q\right)}{\theta\left(X\left(q^{-1 / 2} Z\right)^{ \pm 1} ; q\right)} e^{-\eta \partial_{z}}\right) e^{-\pi i z^{2} / \eta}=0 .
\end{aligned}
$$

Поскольку параметр $X$ является произвольным, выводим искомое равенство. Полученный результат приводит к соотношению $\mathrm{M}(n \eta) \mathrm{M}_{\mathrm{ren}}(-n \eta)=0$ при $n>1$. Для $n=1$ это неверно - из-за наличия сдвигов на $\pm \eta$ тета-функции в знаменателе приводят к появлению полюса на контуре интегрирования в $\mathrm{M}_{\mathrm{ren}}$, следовательно, перестановка интегрирования и действия конечноразностного оператора, использовавшаяся выше, недопустима. Переставляя параметры $\tau$ и $2 \eta$, мы получаем полностью аналогичную картину и для оператора $\mathrm{M}(m \tau / 2)$. Что касается равенств (56), то они, по всей видимости, должны вытекать из (55) после конечноразностного "интегрирования по частям".

Полное пространство $\operatorname{Ker} \mathrm{M}(g)$ весьма велико. Помимо изучавшихся нами нулевых мод оператора $\mathrm{M}(n \eta+m \tau / 2)$ с $n, m \in \mathbb{Z}_{\geqslant 0}$, существуют нулевые моды оператора $\mathrm{M}_{\text {ren }}(-n \eta-m \tau / 2)$. В настоящее время мы не можем полностью описать $\operatorname{Ker} \mathrm{M}(g)$ в компактном виде. Однако мы видим, что можно однозначно охарактеризовать конечномерное пространство нулевых мод, построенных из произведений тета-функций, описанное выше, как пересечение двух инвариантных подпространств:

$$
\text { произведения тета-функций }=\operatorname{Ker} \mathrm{M}(g) \cap \operatorname{Im~}_{\mathrm{ren}}(-g) \text {, }
$$

где

$$
g=n \eta+m \frac{\tau}{2} \quad \text { или } \quad g=\frac{1}{2}+n \eta+m \frac{\tau}{2}, \quad n, m \in \mathbb{Z}_{>0} .
$$


Это утверждение можно также получить, если рассмотреть явный вид оператора (53). Используя формулы (39), можно отфакторизовать зависимость от $z$ в произведение четных тета-функций, входящих в подынтегральное выражение в (53). Следуя обсуждению, приведенному в разделе 5, можно заключить, что оператор $\mathrm{M}_{\text {ren }}(-n \eta-m \tau / 2)$ с $n, m>0$ отображает все пробные функции в произведения четных тета-функций, которые в точности образуют пространство нулевых мод оператора $\mathrm{M}(n \eta+m \tau / 2)$ с $n, m>0$, описанное нами. Дополнительно такое представление оператора (53) должно позволить описать ядро самого оператора $\mathrm{M}_{\mathrm{ren}}(-n \eta-m \tau / 2)$ как пространство функций, для которых подходящее множество “тета-функциональных моментов" обращается в ноль. Это наблюдение требует дальнейшего детального изучения.

\section{9. ЗАКЛЮЧЕНИЕ}

Мы описали конечномерное пространство нулевых мод интегрального оператоpa $\mathrm{M}(g)$, возникающее для двух дискретных решеток спина $g$. В частности, мы получили явное выражение для $\mathrm{M}(g)$, действующего в этом пространстве, в виде конечноразностного оператора. Этот оператор является сплетающим для эллиптического модулярного дубля, и его нулевые моды, заданные в виде произведений тета-функций с двумя различными модулярными параметрами, определяют конечномерные представления этой алгебры. В качестве следующего шага необходимо найти замкнутый вид действия генераторов алгебры Склянина в некотором базисе этого пространства. Здесь необходимо подчеркнуть, что базис можно выбрать произвольным образом, и в некоторых специальных случаях он может ключевым образом упростить ситуацию. Например, произведения тета-функций

$$
h_{k}^{(N)}(w ; p, q):=\prod_{j=0}^{k-1} \theta\left(q^{j} a w^{ \pm 1} ; p\right) \prod_{j=0}^{N-k-1} \theta\left(q^{j} b w^{ \pm 1} ; p\right), \quad k, N \in \mathbb{Z}_{\geqslant 0},
$$

использовались в качестве базисных векторов пространства $\Theta_{2 N}^{+}$в работах [26], [27] для упрощенного анализа эллиптических $6 j$-символов (эти функции впервые возникли как некоторые сплетающие векторы в работе [28]). Для эллиптического модулярного дубля необходимо рассмотреть двухиндексные базисные векторы

$$
h_{k j}^{(N, M)}(w):=h_{k}^{(N)}(w ; p, q) h_{j}^{(M)}(w ; q, p)
$$

и обобщить рассуждения из работ [26], [27] на этот случай. Мы ожидаем, что после подходящего выбора меры можно будет построить двухиндексные биортогональные функции из работы [29].

Полученное в работе [12] тождество $\mathrm{M}(g) \mathbb{R}_{12}=\mathbb{R}_{12}^{\prime} \mathrm{M}(g)$, где $\mathbb{R}_{12}$ является решением уравнения ЯБ и $\mathbb{R}_{12}^{\prime}$ обозначает другой аналогичный оператор, показывает, что ядро оператора $\mathrm{M}(g)$ отображается в себя $R$-матрицей $\mathbb{R}_{12}$. Поэтому нулевые моды оператора $\mathrm{M}(g)$ образуют инвариантное пространство под действием оператора $\mathbb{R}_{12}$. Явный вид соответствующих конечномерных $R$-матриц будет рассмотрен в последующих публикациях.

Благодарности. Работа С. Э. Деркачёва финансово поддержана РФФИ (гранты № 13-01-12405 и 14-01-00341). Работа В. П. Спиридонова финансово поддержана РФФИ (гранты № 11-01-00980, 14-01-00474) и Научным фондом НИУ ВШЭ (грант № 13-09-0133). 


\section{Список литературы}

[1] L. D. Faddeev, "Modular double of a quantum group", Conférence Moshé Flato, v. 1 (Dijon, France, 5-8 September, 1999), Mathematical Physics Studies, 21, eds. G. Dito, D. Sternheimer, Kluwer, Dordrecht, 2000, 149-156.

[2] B. Ponsot, J. Teschner, Commun. Math. Phys., 224:3 (2001), 613-655, arXiv: math/0007097.

[3] S. Kharchev, D. Lebedev, M. Semenov-Tian-Shansky, Commun. Math. Phys., 225:3 (2002), 573-609, arXiv: hep-th/0102180.

[4] A. G. Bytsko, J. Teschner, Commun. Math. Phys., 240:1-2 (2003), 171-196, arXiv: math/0208191.

[5] F. J. van de Bult, Adv. Math., 204:2 (2006), 539-571.

[6] Л Д. Фаддеев, Функи. анализ и его прил., 42:4, 98-104.

[7] R. J. Baxter, Exactly Solved Models in Statistical Mechanics, Academic Press, London, 1982.

[8] M. Jimbo (ed.), Yang-Baxter Equation in Integrable Systems, Advanced Series in Mathematical Physics, 10, World Sci., Singapore, 1990.

[9] Л. А. Тахтаджян, Л. Д. Фаддеев, УМН, 34:5 (1979), 13-63.

[10] Е.К. Склянин, Функи. анализ и его прил., 16:4 (1982), 27-34.

[11] Е.К. Склянин, Функи. анализ и его прил., 17:4 (1983), 34-48.

[12] С.Э. Деркачев, В. П. Спиридонов, УМН, 68:6(414) (2013), 59-106, arXiv: 1205.3520.

[13] В. П. Спиридонов, Алгебра и анализ, 20:5 (2008), 155-185, arXiv: 0801.4137.

[14] В. П. Спиридонов, ТМФ, 139:1 (2004), 104-111, arXiv: math/0312502.

[15] G. E. Andrews, R. Askey, R. Roy, Special Functions, Encyclopedia of Mathematics and its Applications, 71, Cambridge Univ. Press, Cambridge, 1999.

[16] V.P. Spiridonov, S. O. Warnaar, Adv. Math., 207:1 (2006), 91-132.

[17] И. М. Гельфанд, М. И. Граев, Н. Я. Виленкин, Обобщенные функции, т. 5: Интегралъная геометрия и связанные с ней вопросы теории представлений, Физматлит, М., 1962.

[18] A. W. Knapp, Representation Theory of Semisimple Groups. An Overview Based on Examples, Princeton Mathematical Series, 36, Princeton Univ. Press, Princeton, NJ, 1986.

[19] С.Э. Деркачёв, А. Н. Манашов, Алгебра и анализ, 21:4 (2009), 1-94.

[20] S. È. Derkachev, Зап. научн. сем. ПОМИ, 335, 2006, 134-163, arXiv: math.QA/0503396.

[21] S. Derkachov, D. Karakhanyan, R. Kirschner, Nucl. Phys. B, 785:3 (2007), 263-285, arXiv: hep-th/0703076.

[22] В. П. Спиридонов, УМН, 56:1(337) (2001), 181-182.

[23] D. Chicherin, S. Derkachov, D. Karakhanyan, R. Kirschner, Nucl. Phys. B, 868:3 (2013), 652-683, arXiv: 1211.2965.

[24] A. Zabrodin, Internat. Math. Res. Not., 11 (1999), 589-614.

[25] E. Rains, S. Ruijsenaars, Commun. Math. Phys., 320:3 (2013), 851-889, arXiv: 1203.0042.

[26] H. Rosengren, Ramanujan J., 13:1-3 (2007), 131-166.

[27] H. Rosengren, Internat. Math. Res. Not., 60 (2004), 3207-3232.

[28] T. Takebe, J. Phys. A: Math. Gen., 28:23 (1995), 6675-6706.

[29] V.P. Spiridonov, Алгебра и анализ, 15:6 (2003), 161-215, arXiv: math/0303205.

Поступила в редакцию 10.11.2014 